\title{
Machine Vision: Approaches and Limitations
}

\author{
Moisés Rivas López, Oleg Sergiyenko and Vera Tyrsa \\ Engineering Institute of Autonomous University of Baja California, Polytechnic \\ University of Baja California \\ Mexicali, BC, México
}

\section{Introduction}

Machine vision in its common definition is a possibility of a machine (by sensing means and computer mathematic processing consecutively) to obtain an information about surrounding environment for further analytical treatment.

According to this common definition we can unite in a general classification various, sometimes quite different by its principle, technical systems.

These classification tables can be represented on the base of two different approaches: 1) practical causes (Soini, 2001) for necessity to "see surrounding environment", and 2) technical principle and means using for this task solution.

According to the common definition any complete Machine vision system combines two components: technical means (or hardware) and information processing mathematics and algorithm (or software). However, the various software analyses is not expedient in view of variety of mathematical methods and their object focused applications in each case (Mordohai \& Medioni, 2006); and finally can't give clearer problem understanding.

We are now observing a rapid growth of 3D software and hardware capabilities for mainstream PCs, and 3D graphics accelerator boards with processing capabilities of roughly millions polygons per second are becoming commonplace (Petrov et al., 1998). At the same time, dynamic level-of-detail algorithms - built into standard 3D software packages - offer considerable acceleration of model viewing and progressive loading and transmission of 3D models. Despite the fast growth of computer 3D visualization capabilities, until recently data input technology has remained unchanged.

So, in our research for better understanding what is Machine vision, what is its modern state, which practical and technical tasks it decide, and which objective limitations and open problems recently it have, we'll based on the two mentioned above approaches.

In a part of practical reasons, which caused for necessity to develop Machine (or computer) vision concept, can be mentioned:

- $\quad$ security problems in static/dynamic image analysis in perimeter/volume protection (motion/dangerous object detection); (Chellappa et al., 2005), (Itti \& Baldi, 2005)

analysis of short/long term deformation of important engineering structures (more commonly known as 'structural health monitoring' or SHM); (Athavale et al., 1990), (Allen et al., 2005), (Mallet et al., 2004), (Tyrsa et al., 2004), (Ohno et al., 2002), (Benedetti et al., 2004), (Slob \& Hack, 2004), (Stewart \& Tsakiri, 2002), (Liwen Dai et al., 2002) 
- $\quad$ surface digital mapping and micro-surface analysis; (Winkelbach et al., 2006), (Slob \& Hack, 2004), (Tunstel, 1995), (Vang et al., 2000), (Peng-Hsiang Weng \& Fu-Jen Kao, 2004)

- automatic navigation of robot in unknown scene (Tunstel, 1995), (Diosi \& Kleeman, 2005), (França et al, 2005), (Ibeanusi et al, 1999), (Chaumette \& Hutchinson, 2006), (Surmann et al, 2003), (Kee et al, 2008), (Hada \& Takase, 2001)

In a part of technical principle and means Machine vision system can be classified to:

- camera (or "stereo camera") principle with further image analysis algorithms; (Chellappa et al., 2005), (Itti \& Baldi, 2005), (Selectes papers on CCD and CMOS imadges, 2003), (Lavelle et al., 2004)

- 2D-3D image reconstruction techniques (most frequently - 3D laser scanning on triangulation principle); (Tyrsa et al., 2004), (Peng-Hsiang Weng \& Fu-Jen Kao, 2004), (França et al, 2005), (Surmann et al, 2003), (Lavelle et al., 2004), (Handbook of Optical and Laser Scanning, (2004), (Forman \& Parry, 2001), (Pierce et al., 1992), (Lichti et al., 2000), (Petrov et al., 1998)

- terrestrial laser total stations, or airborne laser scanners; (Slob \& Hack, 2004), (Baltsavias, b, 1999), (Baltsavias, a, 1999), (Wehr \& Lohr U, 1999)

- obstacle detection y description techniques, based on signal "time-of-flight" (radar, lidar, sonar, rangefinders or UWB technologies); (Vang et al, 2000), (Pierce et al., 1992), (Yu et al., 2008)

- GPS-based systems for objects surface reconstruction; (Kee et al., 2008), (Stewart, \& Tsakiri, 2002), (Hada \& Takase, 2001), (Liwen Dai et al., 2002)

- combined systems, which use a certain combination of mentioned above basic means for increase total system resolution and noise robustness (Ohno et al., 2002), (Benedetti et al., 2004), (Lavelle et al., 2004), (Retscher, 2007), (Liu et al., 2006).

The goal of our work is to compare several of mentioned approaches to Machine vision design, compare their advantages over each other, and the most principal limitations for practical application.

In general, variety of practical applications which strongly requires the technical vision device it is just a confirmation of an actuality and high importance of a new technical vision methods development.

However, sometimes very different practical tasks use very similar techniques for practical task solution. And it has a different level of success. Because of various practical limitation and specific requirements which appears in each unique case. So, the key parameter for to analyze different technical principle for machine vision system design is a basic technical device for task solution.

\section{Approaches to machine vision design}

More attentive analysis of the mentioned above technical principle and means list permit us to make a simplified conclusion. There are four completely distinct technical approaches to technical vision device design. More truly, if to be rigorous in definitions, three relatively independent methods, and the fourth group which cannot be an independent basis for creation of the device, but possesses such important advantages, that at use of other methods it is not to forget about them, and it is desirable to use actively them as auxiliary means.

These four groups are: 
- camera methods;

- laser scanning systems;

- GPS-based methods;

- numerous rangefinder devices (radar-, sonar- , laser-principle, etc.)

As evident the last one cannot be an independent basis for creation of the complete Machine vision system. Because of its physical nature this system it is only capable to estimate the distance to "averaged object", but not reconstruct its surface point-by-point.

Let us carefully review all mentioned principles of Machine vision design for establish their strong and weak points.

\subsection{Camera based machine vision}

The machine vision system includes (Selectes papers on CCD and CMOS images, 2003) a stereo TV camera assembly and a processing unit. It detects obstacles in real time within its field of view in a range from $5 \mathrm{~m}$ to $50 \mathrm{~m}$ ahead of the vehicle with a viewing angle of 40 degrees. The cameras are arranged vertically at the front part of the vehicle. The system locates obstacles in the trapezoidal field of view. The scanning of each camera is synchronized and the processing unit uses hard-wired logic instead of a programmable device in order to realize high speed processing of video signals from the cameras. The principle of the obstacle detection is parallax. When two images from both of the cameras are compared, the two images of an obstacle are identical except the positions in the frames. On the other hand each image of figures on the ground differs due to the positions of the cameras. Fig. 1 illustrates the principle of the obstacle detection.

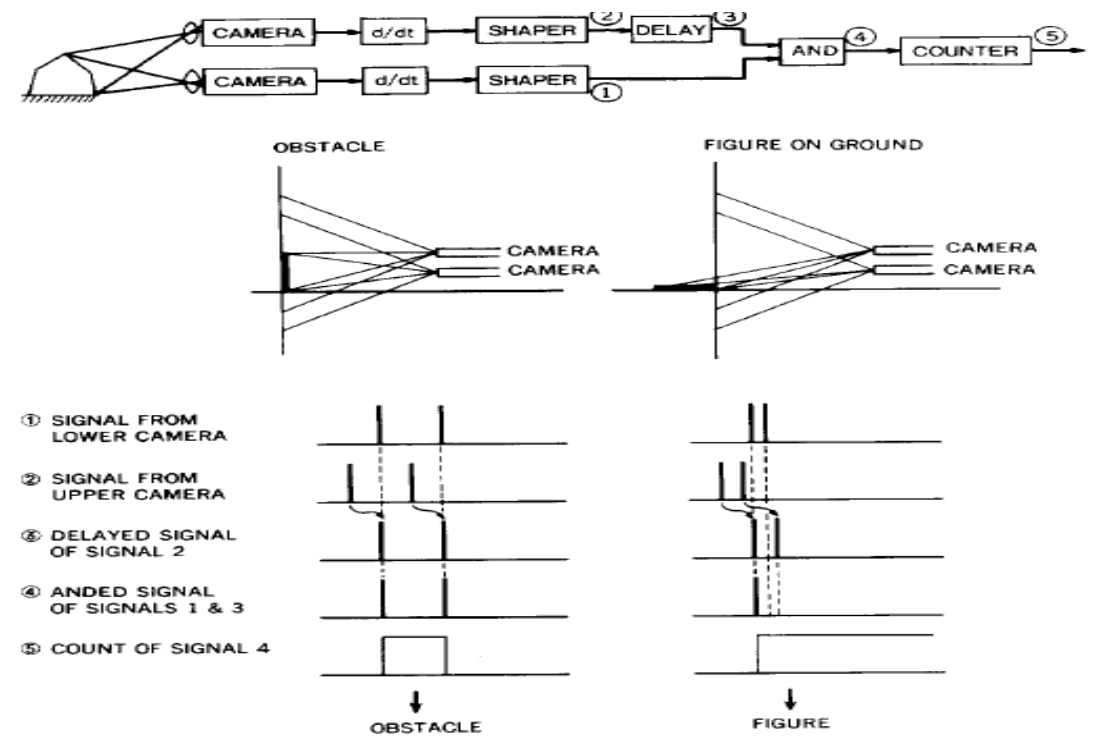

Fig. 1. The principle of the real time obstacle detection

The video signals are differentiated regarding time and the signals are shaped to obtain pulses that correspond to edges in the images. Each time interval of the pulses from each cameras, (signal 1 and signal 2 in Fig. 1), discriminates an obstacle from a figure on a road. 
An obstacle generates same time intervals, but a figure on a road generates different time intervals. The cameras have to be, thus, synchronized with each other, and have to employ vertical and progressive scanning techniques. The position of a scanning line corresponds to the direction to the obstacle, and the point where the optical axes of the cameras are crossing indicates the distance to the obstacle. Delaying of one of the signals from the TV cameras is equivalent IO rotation of the optical axis of the camera. Thus, varying the delay time enables us to detect obstacles at other locations. For enlargement of the field of view and detection of obstacle:; in the two-dimensional field of view during one scanning period, parallel processing with 16 kinds of delay time is employed, which yields the field of view of 16 zones arranged longitudinally at intervals of $\mathbf{1}$ m. Time required to detect obstacles is 35.6 ms, which consists of $33.3 \mathrm{~ms}$ of scanning OF one frame and $2.3 \mathrm{~ms}$ of processing to detect and locate obstacles. Fig. 2 shows an example of the obstacle detection. The guardrail is identified as a series of obstacles that are indicated by black elements in the figure at the bottom. Since the system had no measures against brightness, shadows, and shades, the operating condition was restricted.

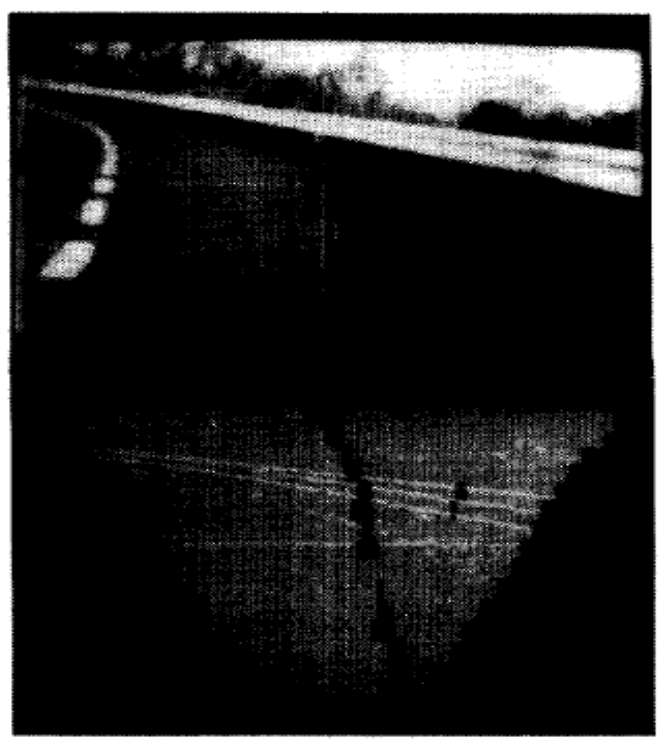

Fig. 2. The obstacle detection: a road scene (top) and obstacles in the scene (bottom) (Sadayuki Tsugawa, 1994)

In a basis of any camera method it is the principle of the human volumetric vision, capable to reconstruct a 3-dimensional picture and approximately estimate distances up to the objects within scene. That is in other words, stereovision.

Any stereovision technical system is approach to a multicamera system. In the elementary case under consideration it is two cameras system. If a stereovision system (Chaumette \& Hutchinson, 2006) is used, and a 3-D point is visible in both left and right images (see Figure 3 ), it is possible to use as visual features s (vector $\boldsymbol{s}$ contains the desired values of the scene/features): 


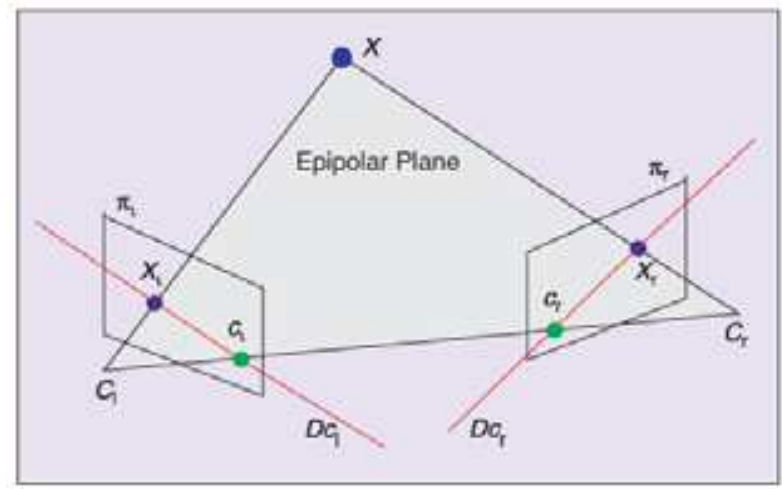

Fig. 3. A stereovision system

$$
\mathbf{s}=\mathbf{x} s=(\mathbf{x} l, \mathbf{x} r)=(x l, y l, x r, y r),
$$

i.e., to represent the point by just stacking in $\mathbf{s}$ the $x$ and $y$ coordinates of the observed point in the left and right images.

For a 3-D point with coordinates $X=(X, Y, Z)$ in the camera frame, which projects in two images as a 2-D point with coordinates $\mathbf{x}=(x, y)$, we have (Chaumette \& Hutchinson, 2006):

$$
\left\{\begin{array}{l}
\mathrm{x}=\mathrm{X} / \mathrm{Z}=\left(\mathrm{u}-\mathrm{c}_{\mathrm{u}}\right) / \mathrm{f} \alpha \\
\mathrm{y}=\mathrm{Y} / \mathrm{Z}=\left(\mathrm{v}-\mathrm{c}_{\mathrm{v}}\right) / \mathrm{f},
\end{array}\right.
$$

where image measurements matrix $\mathbf{m}=(u, v)$ gives the coordinates of the image point expressed in pixel units, and $\mathbf{a}=(c u, c v, f, \alpha)$ is the set of camera intrinsic parameters: $c u$ and $c v$ are the coordinates of the principal point, $f$ is the focal length, and $\alpha$ is the ratio of the pixel dimensions. In this case, we take $\mathbf{s}=\mathbf{x}=(x, y)$, the image plane coordinates of the point.

Taking the time derivative of the projection equations (1), we obtain the result which can be written in general form

$$
\mathbf{X}=\mathbf{L}_{\mathrm{x}} \mathbf{V}_{c}
$$

where $\mathbf{V}_{c}$ is a spatial velocity of the camera be denoted by $\mathbf{v}_{c}=\left(v_{c}, \omega_{c}\right)$, (with $v_{c}$ the instantaneous linear velocity of the origin of the camera frame and $\omega_{c}$ the instantaneous angular velocity of the camera frame) and the interaction matrix $\mathbf{L}_{x}$ (we consider here the case of controlling the motion of a camera with six degrees of freedom) related to $\mathbf{x}$ is

$$
L_{x}=\left[\begin{array}{cccccc}
\frac{-1}{\mathrm{Z}} & 0 & \frac{x}{\mathrm{Z}} & \mathrm{xy} & -\left(1+x^{2}\right) & y \\
0 & \frac{-1}{\mathrm{Z}} & \frac{y}{\mathrm{Z}} & 1+y^{2} & -x y & -x
\end{array}\right]
$$

$L_{\mathbf{x}}$ is an interaction matrix related to $s$, or feature Jacobian. In the matrix $\mathbf{L}_{\mathbf{x}}$, the value $Z$ is the depth of the point relative to the camera frame. Therefore, any control scheme that uses this form of the interaction matrix must estimate or approximate the value of $Z$. Similarly 
(Chaumette \& Hutchinson, 2006), the camera intrinsic parameters are involved in the computation of $x$ and $y$.

Let us analyze this basic "camera method" parameters regard to equations (1)-(3).

1. Focal distance $f$ in (1), as well as the set of camera intrinsic parameters, shows us that camera uncertainty is strongly related to fabrication imperfections for each unique camera. And, hence the absolute value of this camera uncertainty is rising sufficiently with scene depth increase (proportionally to $1 f, 2 f \ldots n f$ ).

2. $\quad \mathbf{V}_{c}$ in (2), which is camera velocity, shows us that any camera method error strongly related to any own camera motions. And, taking to account an arbitrary character of camera self-motions and consequently instant vector $\mathbf{V}_{c}$ direction, it is very difficult to estimate real camera uncertainty.

3. Depth $Z$ in (3) shows for components $(1 ; 1),(2 ; 2)(2 ; 3)$ for example, that method resolution and reasonable operating rate are strongly limited by own theory.

These reasons permits us to understand exactly clear a principal limitations for any practical application of camera based technical vision method. They are very critical for practical using in any application with self-moving camera positioning, are extremely sensible to any vibration and dynamic shocks. Moreover, in spite of significant achievements of camera technologies in last ten years, it still exist a possibility of "camera error", i.e. when one typical object image is assigned with typical properties of another. And this possibility is proportionally increased with distance growth. Finally, camera methods application is naturally limited with distances up to $50 \mathrm{~m}$.

\subsection{Laser principle based machine vision systems}

Modern laser sensor technologies have made fast and accurate 3-D data acquisition possible as evidence by several commercialized 3-D laser sensors (Tyrsa et al., 2004), (Peng-Hsiang Weng \& Fu-Jen Kao, 2004), (Diosi \& Kleeman, 2005), (França et al., 2005), (Surmann et al., 2003), (Lavelle et al., 2004), (Handbook of Optical and Laser Scanning, 2004), (Forman \& Parry, 2001), (Pierce et al., 1992), (Lichti et al., 2000), (Petrov et al., 1998). Other than some existing 3-D technologies based on CCD 2-D image reconstruction, these 3-D sensors are based on laser scanning and geometric methodologies such as triangulation (Petrov et al., 1998), (Tyrsa et al., 2006, a), (Tyrsa et al., 2006, b), (Rivas et al., 2008) and have achieved more and more attentions in machine vision application due to its robustness and simplicity. However, to expand functional application of these laser sensors, powerful algorithm addon is still needed to effectively process measured data from them, normally depending on individual application case.

Laser triangulation principle (Handbook of Optical and Laser Scanning, 2004) in general can be based on two schemes represented in Fig 4 ( $a$ and b). The first one uses a fixed angle of emission and variable distance; the second one, on the contrary, fixed triangulation base and variable scanning angle.

The first one works as follows.

A laser beam is projected onto the measurement surface (Fig. 4, a), where it is scattered from the surface and its image is detected by an optical detector, usually a CCD camera. By using a suitable angular arrangement between the laser and sensor positions, the detected location of the laser spot on the image plane produces an accurate measurement of the distance between the sensor and the surface. Therefore, the profile of a surface can be measured by using laser triangulation. The laser beam is made to scan across the surface of the object. The 
range data at each location is calculated according to its position within the image plane so that the whole 3-dimensional profile of the surface can be obtained. The positioning of the laser beam is normally controlled by an adjustable mirror system, which is able to change the angular direction of the laser beam over a 2-dimensional plane.

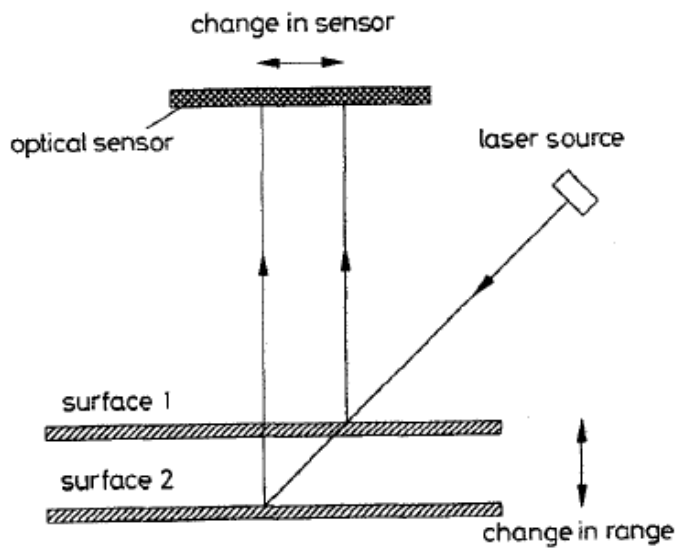

a)

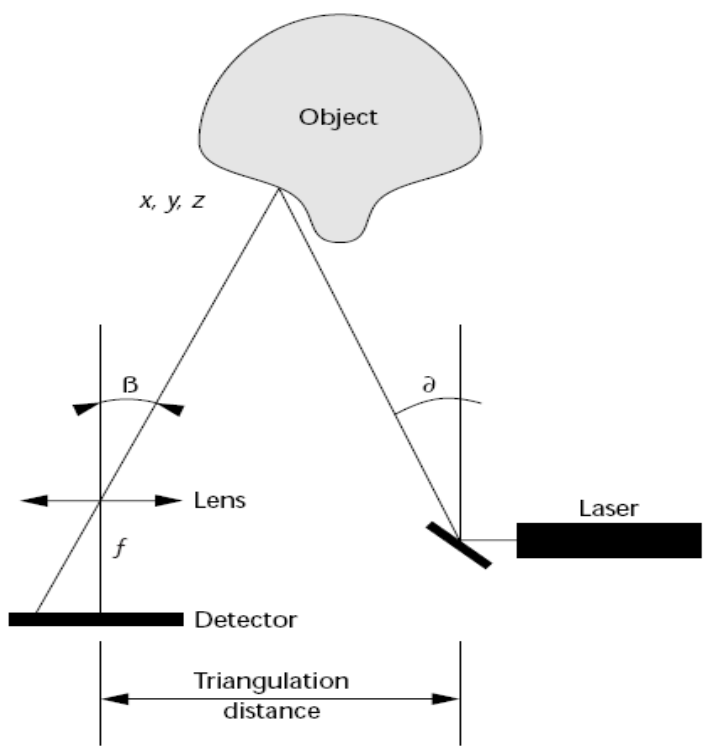

b)

Fig. 4. Two principles of laser triangulation (a - "with fixed angle of emission"; b - "with fixed triangulation distance", consists of a laser dot or line generator and a 1D or 2D sensor located at a triangulation distance from the light source (Petrov et al., 1998). Optionally, the laser light can be steered by a motor) 
A second one basic triangulation scheme (Fig. 4, b) is an active optical triangulation 3D digitizing systems, which visualize real-life objects. These active optical systems provide photorealistic representations of shapes and textures with reasonable speed. Figure $4, \mathrm{~b}$ shows a simple diagram of a triangulation scanner. The laser beam-reflected from a mirror-is projected on the object. The diffusely reflected light is collected by the sensor, which is a linear array if a laser dot is projected or a 2D matrix (typically a charge coupled device camera) if laser stripes are projected.

The laser positioning circuitry controls the angle 0 and is known. The angle $\beta$ is determined in the sensor measurements if the focal length $f$ and the detector pixel size are given. The triangulation distance - the distance between the sensor and the mirror - is also known.

As Figure 4, b shows, since all geometric parameters are known, the $x, y, z$ coordinates of the point on the object can be computed in a trigonometric fashion. If a single laser dot is projected, the system measures the coordinates of just one point of the object. When a laser stripe is projected, all points along the stripe are digitized. The basic triangulation scheme can be enhanced to improve the optical quality and depth of field (see www.vit.iit.nrc.ca for several good examples). The modifications, however, require custom-manufactured components and are relevant mostly for scanners used in high-accuracy reverse engineering tasks. In general, any other kind of structured light can replace the laser dot or stripe. For example, several dots or laser stripes can be projected. However, if the system projects multiple patterns, it's difficult to identify individual elements. If, say, two stripes are projected, then the image processing software must separate the first and second lines. Solutions to the identification problem include using a sequence of different colored stripes. Such schemes typically become sensitive to ambient light, as we'll discuss later.

The most well-known optical triangulation 3D scanner is the one developed by Cyberware of Monterey, California. This legendary scanner was used by a generation of computer scientists. The company has maintained essentially the same product line for more than 10 years. Cyberware products can capture photorealistic images of a range of objects - from apple-size models to fullsize human bodies. The scanner head contains a laserline generator, a system of mirrors, and black-and-white and color video cameras. Scanning occurs by moving the object on a rotation and translation platform, or by moving the sensor around the object in a circular motion. In the basic Cyberware scanner model, a system of mirrors collects laser light from left and right triangulation paths relative to the laser. This scheme helps avoid shadows in the scans caused by the triangulation angle. However, it imposes strict requirements on the optical assembly's quality and the system's calibration, and increases the scanner's size. The scanners are complex, not portable, and prohibitively expensive for many applications. One version, the full-body scanner, digitizes a complete human body as a combination of four scans in about 17 seconds. Each scan has $250 \times 1000$ points of resolution. The four scans can be glued using commercial software packages (Petrov et al., 1998).

Another typical method for laser scanners is a Circular laser scanning vision sensor for detecting position of singular points (Tao Dai et al., 2005). Usually it called data acquisition device-circular scanning sensor (CSS). By singular point in this case we mean small convex or concave points deviated from smooth surface manifold, caused by either faulty or normal production/machining. The platform for this devise features a three degree of freedom (hence, accommodate 3-D) motion control through a processor, three servo motors and a circular scanning sensor (CSS), as well as a computing system. An emulated head is 
attached rigidly with CSS to indicate the tracking and focusing actions. The circular scanning sensor (CSS) (Figure 5), with embedded data acquisition device, is a laser scanning multi-axis machine vision device which produces 3-D data sequences of a target work piece for reconstruction of geometry characteristics.
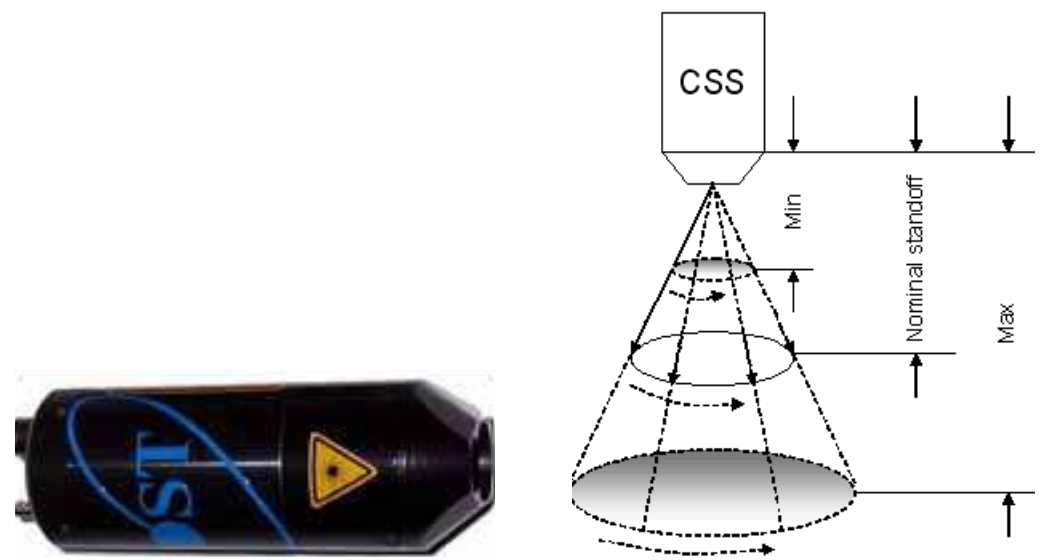

Fig.5. A Laser Circular Scanning Sensor and illustration of Circular Scanning Principle

The CSS and the emulated head are driven by three servo motors to generate 3-D $(x, y, z$ axis) motion: arbitrary planar scanning route, up and down motion. The main emulated head could track any 3-d singular point on the surface of the work piece while conducting scanning and concentrate on it at specified focusing distance.

The motors are controlled by a central processor which receives location information of singular points from a PC system. The loop is then closed by feedbacking 3-D geometric data of target points acquired by the CSS to the PC system which processes the data sequence continuously using DMA based algorithm (Fig. 6.) to return the location information of singular points, if any, on the surface of work pieces.

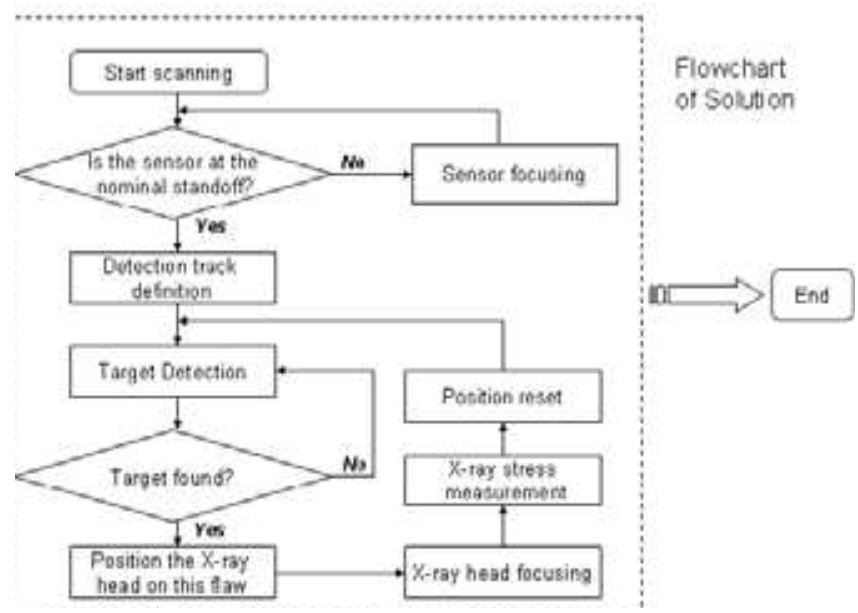

Fig. 6. Flow-Chart of DMA Based Operating Algorithm 
For central laser scanning sensor, scanned data is obtained in sequence:

$$
X i=\left[x_{0}, x_{2}, \cdots, x_{N-1}\right], i=1,2, \cdots,
$$

where $X i$ indicates the $i-t h$ data sequence captured and $N$ is the number of scanning times called the depth of the data sequence. This data sequence could represent data piece acquired by one sensor in different pre-set time period or by multiple sensors at same time. For example, a line or circular scanning laser sensor generates a geometric data sequence of objects in one scanning cycle. For circular scanned data is considered, hence the depth $N$ is also the scanning cycle period. An example of a 1-D real circular scanning data cycle is illustrated in Figure 7, which is conducted, for example, on a horizontally flat iron plate placed on a table.

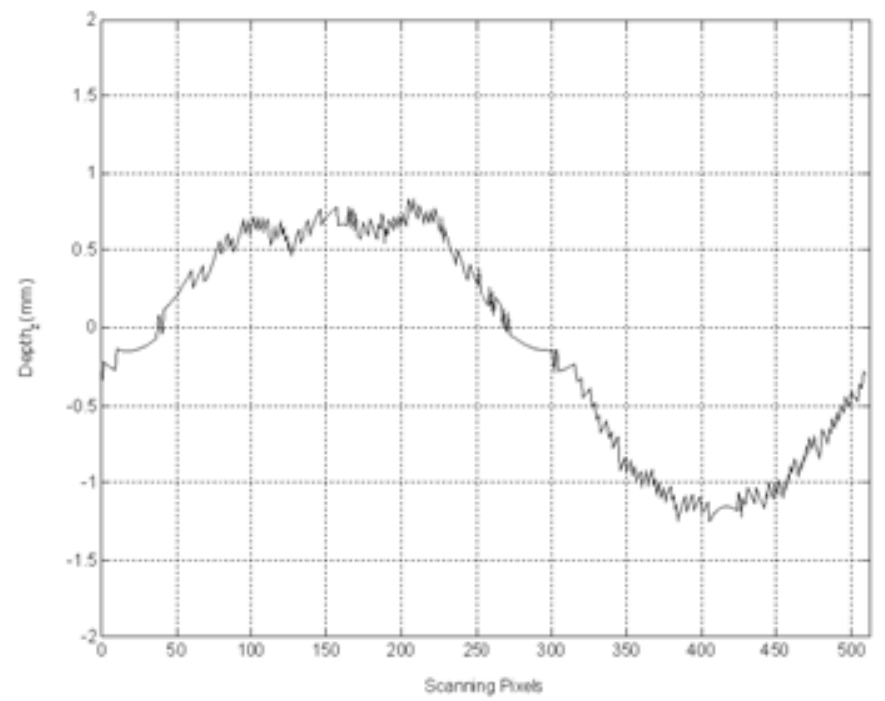

Fig. 7. A 1-D Circular Scanning Data Cycle

In ideal case, one would expect scanned data yields a horizontal line. However, this hardly happens in reality. Instead, as Figure 7 shows, a pseudo-sinusoid shape is presented reflecting either unevenness of plate surface or table leaning, or both. In the case that there is a hole-like singular point, caused by either normal or faulty machining, on the surface, the scanned data would normally reflect that point with a peak significantly deviated from the profile, unlike small peaks around the profile induced by noise. Such an example with a hole of depth $0.4 \mathrm{~mm}$ at the 100th pixel is shown in Figure 8 (1-D circular scanning data).

From these two examples, it is clear that two major tasks have to be performed in order for us to obtain accurate information of targets from laser scanned data: reduction of effect of noise on the measurement and detection of singular peak.

This method even it is a laser scanner practically is similar to 3-D technologies based on 2-D image reconstruction and posses all weak points, which are mentioned for these group of methods.

For static object shape monitoring also possible to use a multi-beam laser triangulation (Noll \& Krauhausen, 2003) up to more than 120 laser beams are used to simultaneously measure geometric features of moving products such as e.g. the thickness, profile or surface topology. The laser beams form a series of measuring points or lines on the object surface. 


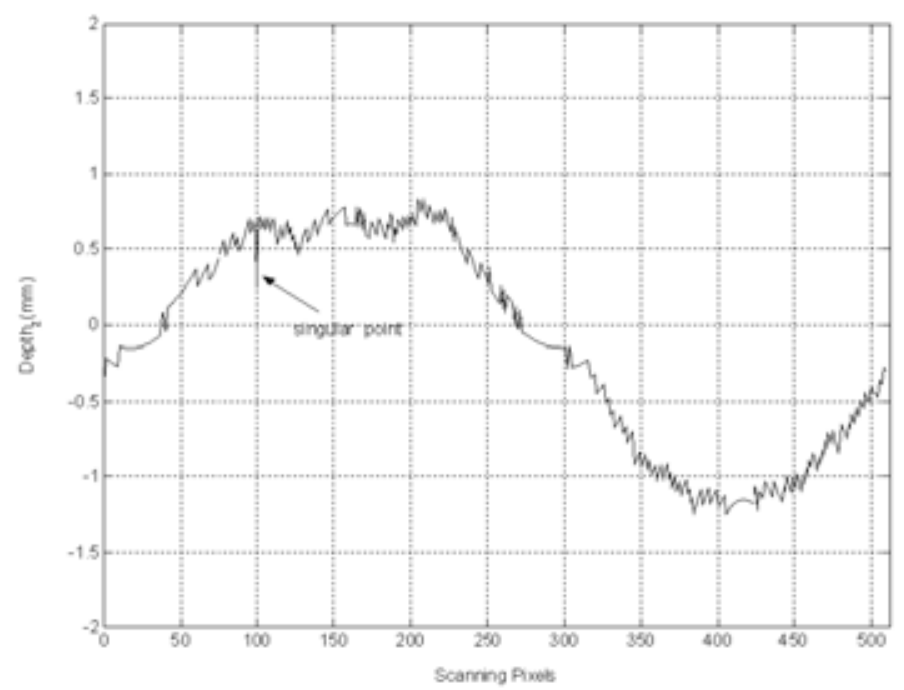

Fig. 8. A 1-D Circular Scanning Data Cycle with a Singular Point

By temporal modulation of the pulse width of the laser diodes and control of the exposure time of the CCD or CMOS detectors a dynamic range of more than $1 \div 10^{4}$ (without considering the dynamic range of the detector elements itself is realized enabling the measurement of object surfaces ranging from black to metallic bright. The use of different laser wavelengths allows suppressing cross-talking originating from overlapping laser projections on the surface of the specimen. However, this method is a good tool for dynamic surface control, but it is unsuitable for high-grade image reconstruction. It cannot be related to pure "camera methods" because CCD or CMOS devices are used only like "light 1/0 detectors", also for incident ray preliminary positioning. This example highlights those sometimes optical sensors matrixes are the better detectors because of their good sensitivity/resolution.

Another laser tools for static perimeter control is Laser radar (LADAR) (Sahba et al., 2006), (Stutz, 2005). LADAR scanning is far advanced over conventional active infrared (AIR) sensing. The transmitter and receiver units are usually coaxially located, making the installation less prone to misalignment or tremors than traditional opposed or retroreflective sensor posts. Laser scanning also has much finer resolution that RADAR and microwave-RADAR, allowing the definition of the intruding target size. Time of flight pulse detection or continuous wave phase shift methods are used to derive range measurements. According to tests conducted to determine the feasibility of using laser scanning for perimeter protection (Sahba et al., 2006), it has been confirmed the capability of detecting humans and vehicles, with a maximum range of $25 \mathrm{~m}$ and $80 \mathrm{~m}$ respectively. The testing demonstrated large area coverage, the ability to determine intruder size, the definition of multiple detection zones, a good detection rate and low false alarm rate (Hosmer, 2004). It has also been claimed that camouflage and other methods for masking an object can deceive near infrared devices, but it is impossible to mask a physical volume moving through space, thereby implying that by scanning a plane at high speed and resolution, any object moving 
through this plane will be detected (Hancock et al., 1998). Riza, et. al. propose generic criteria for an ideal optical scanner including a wide scanning range, no moving parts, high resolution, high speed and low power consumption. Additionally, they place significance on reconfiguration (Riza \& Muzammil, 2003). By projecting many spots, a laser curtain is produced and multiple range measurements from a dense array of laser spots provide a 3D contour of the perimeter. In current LADAR scanners, a laser beam is deflected by rotating mirrors as show in Fig. 9 where $\mathrm{D}$ is the incident beam diameter, $a$ is the beam-feed angle, $\theta$ is the active scan angle and $\mathrm{L}$ is the scan length.

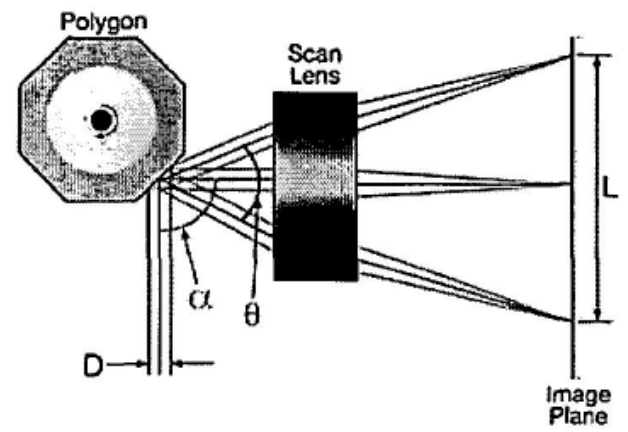

Fig. 9. Principal of laser beam deflection using a rotating mirror (Stutz, 2005)

As we can observe, commonly static object monitoring methods strongly related to basic optical property of focal distance and its practical consequence of a sharp image in a focal plane. From the other hand, it makes these technical solutions initially (apriori) unsuitable for such dynamic task like a mobile robot vision.

In applications where information from more than one physical point of an object needs to be derived, a scanning mechanism must be employed to deflect the light beam from the laser source, towards the desired point of interest on the object surface. This deflection is usually driven in two or three dimensions electro-mechanically. Laser scanning mechanisms have been used in a large variety of applications ranging from photo and document scanners to airborne topographic mapping, surveying of buildings and plants, counting and collision prevention. Approximately over the past ten years laser scanning has found new applications in photoelectric security sensing. Some common uses include providing light curtains in front of assets on a wall display or over the facade of a building. More commonly, laser scanners are employed to provide high resolution range measurements to the outer perimeter (e.g. fencing, walls) of a building and detect a change in range values when beams are intersected by an intruder.

To date, driving the deflection mirror in the desired direction has been accomplished using different devices such as electro-mechanics, rotating polygon shaped mirrors and galvanometers. Figs. 10. (c) and (d) show the layout of a typical commercially available laser scanner and its mirror driving mechanics. Typically, the whole optical head is rotated by a servo motor head around the azimuth in the order of a $1000 \mathrm{rpm}$, and the mirror rotates in elevation at the same time. This dual movement deflects the beam in the desired directions. However, the intrinsic motion of the mirror causes scanning problems which are the hardest to quantify and correct. Acceleration time taken to reach the constant scanning speed from a stationary position can result in range measurements being stored at the wrong corresponding points in the scanned image. Additionally, all mirrors and measurement 
components must be synchronized exactly. In general, laser scanning is much more sensitive to vibration than a multi-beam stationary optic approach. In particular, mirror device scanners are slow, bulky and expensive and being inherently mechanical they wear out as a result of acceleration, cause deflection errors and require regular calibration. Stutz in (Stutz, 2005) explains that the performance of polygonal scanners, especially with respect to maintaining an accurate deflection beam path, is prone to manufacturing tolerances.

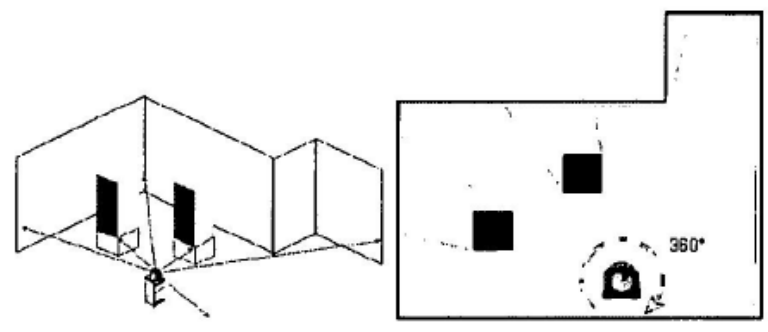

(a)

(b)

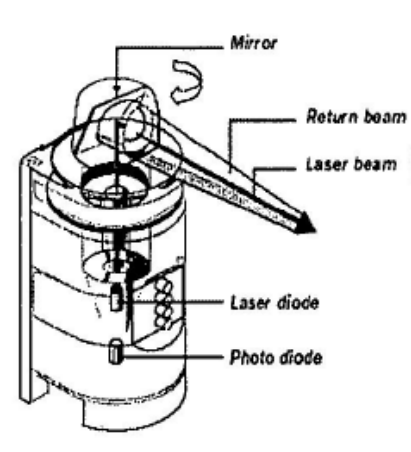

(c)

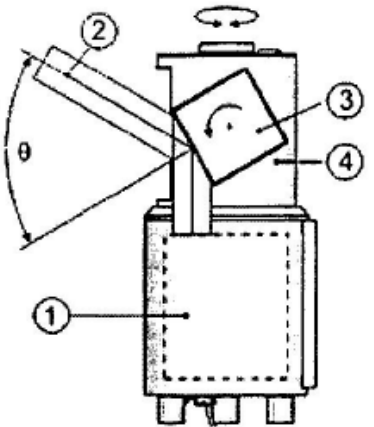

(d)

Fig. 10. (a) and (b) indoor room surveillance scanning, (c) scanner with mirror mounted on motor, (d) scanner with rotating mirror where 1 contains the range finding electronics, 2 is the deflected beam, 3 is the rotating mirror and 4 is the complete optical head which also rotates (Sahba et al., 2006)

Dynamic track and jitter errors are caused by tolerances for:

- Polygon machining errors

- Mounting errors

- Mounting-hub errors

- Random wobble caused by ball bearings

- Motor cogging

- Torque variations

- Noisy start of scan circuit

Machining and mounting errors directly cause the deviation of the deflection beam from the desired path of propagation towards the correct point at the image plane. Errors can range from 1 to 60 arc-seconds in angular deviation. Cogging, torque and facet flatness variations cause errors in the actual scan line. 
Other problems listed with rotational scanners are as follows (Stutz, 2005):

- Synchronization with other time-dependent elements in the system is rather difficult.

- Motor stability and durability at higher rotation speeds also present problems.

- There is an upper limit to the rotation speed due to the tensile strength of the mirror material. The mirror must not disintegrate at the maximum rotation speed.

Another strong tool for 3D spatial data acquisition is terrestrial laser scanning (Slob \& Hack, 2004), (Baltsavias, 1999, a), (Baltsavias, 1999, b). These are active scanners based on laser signals for measurement of slant distances to acquire information about the surface of the Earth and objects on it. As mentioned above, there are several laser scanning systems being operational. Concerning the measurement principle two different methods had been realized: runtime measurement using pulsed laser signals and phase difference measurement using continuous-wave lasers. Because most systems are based on the first mode, it will be described in more detail in the next section. A good overview on both topics can be found in (Wehr \& Lohr, 1999). The pulsed mode laser scanner emits pulsed laser light in exactly determined time intervals. The system measures the runtime of these laser pulses, i.e. the elapsed time between emitting a signal and receiving it after reflection on the surface of the Earth or objects on it. Therefore, slant distances can be derived from these time differences by the wellknown formula $\mathrm{v}=\mathrm{Ds} / \mathrm{Dt}$ or Ds $=\mathrm{v} / \mathrm{Dt}$. By means of the exterior orientation of the sensor (recorded by differential GPS (dGPS) and INS systems) 3D co-ordinates of the illuminated surface points can be determined.

Laser systems need to be designed mainly regarding two components: the emitting and receiving unit and the positioning unit. Both will be described as the example by means of the operational system TopoSys (Wehr \& Lohr, 1999) which was used to acquire the data sets about terrestrial landscape.

In this system, the emitting and receiving unit is realized by means of a glass fiber optic. The laser light is emitted on a nutating mirror, i.e. a rotating mirror which deflects it on a glass fiber bunch. The ends of the glass fibers are connected to a row-shaped optic, so the resulting measuring pattern on the surface of the Earth is a single scanning line. In addition to the movement of the airplane this results in a strip-wise data acquisition as shown in Figure 11.

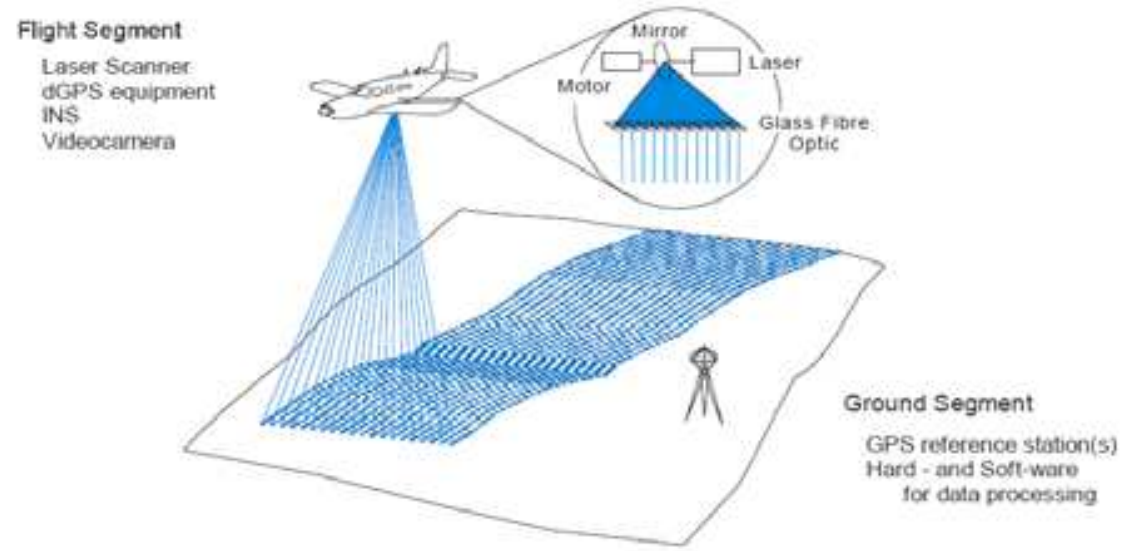

Fig. 11. Laser scanner system TopoSys (Wehr \& Lohr, 1999)

The positioning component of the system consists of several elements. As navigation principle differential Global Positioning System (dGPS) is chosen. Therefore, GPS antennas 
are mounted on the airplane, as well as on reference stations on the ground. Although this positioning strategy yields to good results concerning the accuracy of the obtained coordinates (in the range of some few centimeters), the measurement rate is lower than the one of the laser scanner. Therefore, additionally Inertial Navigation Systems (INS) are used, i.e. navigation units register the rotations of the airplane based on gyros. These are capable to determine a position with a higher temporal resolution.

In Table 1 the performance parameters of this system are listed. It should be mentioned that the system is capable of acquiring up to 5 points per $\mathrm{m}^{2}$. This results in data sets of high point density and suitable accuracy in position as well as in elevation.

\begin{tabular}{|l|l||l|l|}
\hline sensor type & pulse modulated laser Radar & range & $<1000 \mathrm{~m}$ \\
\hline scanning principle & fibre optic line scanner & transmitter & solid state at $1.5 \mathrm{~mm}$ \\
\hline measurement principle & run-time measurement & scan frequency & $300 \mathrm{~Hz}$ (adjustable) \\
\hline field of view & $+1.7^{\circ}$ & number of pixels per scan & 127 \\
\hline $\begin{array}{l}\text { swath width } \\
\text { (1000m flight height) }\end{array}$ & $250 \mathrm{~m}$ & $\begin{array}{l}\text { accuracy of a single distance } \\
\text { measurement }\end{array}$ & $<0.3 \mathrm{~m}$ \\
\hline $\begin{array}{l}\text { accuracies of point } \\
\text { cocrdinates x.y.z }\end{array}$ & $* 0.3,0.3,0.1 \mathrm{~m}$ & $\begin{array}{l}\text { resolution of a distance } \\
\text { measurement }\end{array}$ & $<0.1 \mathrm{~m}$ \\
\hline
\end{tabular}

Table 1. Performance parameters of TopoSys laser system (Steinle \& Vogtle, 2000)

It is relatively more expensive method over another laser application. Hence the principle limitation of it is the practical importance of the task. However, terrestrial laser scanning have a significant flexibility of provided practical use acts. For example, terrestrial scanning lidar (light detection and ranging) is applied to outcrop stratigraphic mapping enables researchers to capture laser range data at a rate of thousands of individual X, Y, Z and laserintensity points per second (Bellian et al., 2005).

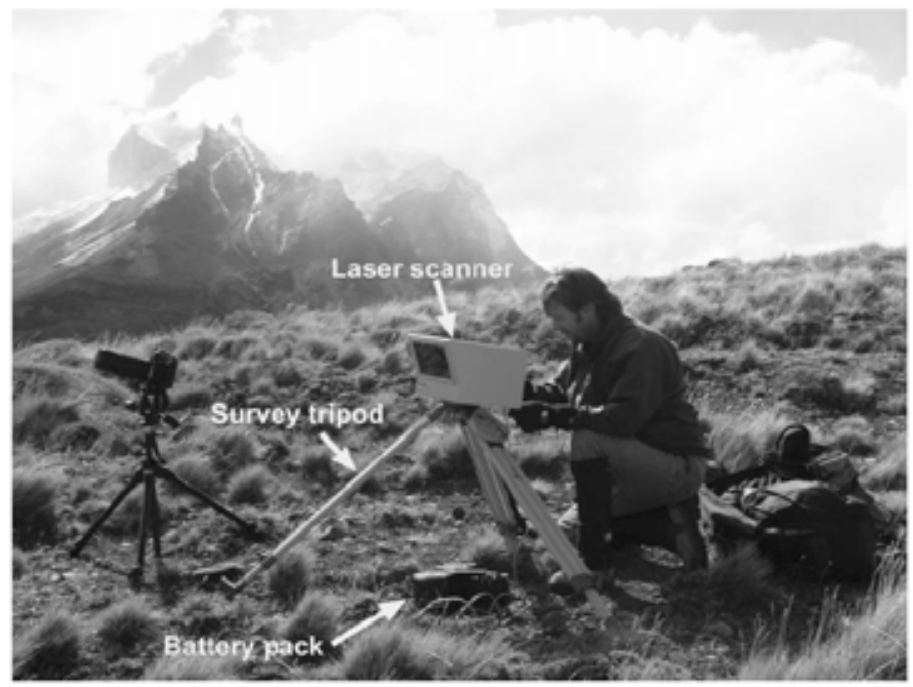

Fig. 12. A typical field setup in Patagonia in early 2002. All equipment was transported in a backpack by a single person (picture courtesy of Jeremy Willson, Shell Oil). Lithium Ion batteries have helped reduce battery weight to 1,540 grams (3.4 pounds) per approximately 2 hours of continuous scan time (Bellian et al., 2005) 
Research group of (Bellian et al., 2005) store the 3D coordinates data set with an Optech Laser Imaging ILRIS 3D terrestrial (ground-based) scanning lidar (Fig.12). This instrument was chosen for its $1 \mathrm{~km}$ range, palm-type interface, and light weight. All data from the ground-based lidar surveys were processed on a standard laptop computer with at least a gigahertz processor speed, 1 gigabyte of RAM and Innovmetric Incorporated's Polyworks CAD (Computer Aided Design) software. The principal result of (Bellian et al., 2005) was: high-resolution lidar (approx. $1 \mathrm{~cm}$ point spacing) is far more accurate in three dimensions than nearly all GPS currently available to the public.

\subsection{GPS-based and combined machine vision principles}

GPS-based solutions sometimes also are acceptable for machine vision, especially for navigation tasks. The good example of such system is presented in (Vang et al., 2000).

GPS based navigation for autonomous land vehicles has the capabilities of GPS to determine the locations of vehicles, as far as static objects, basing on satellites (Kee et al., 2008), (Stewart \& Tsakiri, 2002).

GPS measurements consist of biased and noisy estimates of ranges to the orbiting satellites. The principal source of bias is the unknown receiver clock offset, whereas the remaining errors arise from:

- ' modelling of the satellite clock and ephemeris.

- ' modelling of the ionospheric and tropospheric delay.

- ' measurement of the code and carrier phase influenced by both receiver noise and multipath.

DGPS is a technique that improves the user's position accuracy by measuring the infinitesimal changes in variables in order to provide satellite positioning corrections. It should contain a reference station, a data-link, and user applications. The reference station generates corrections by means of a measured pseudo-range or carrier-range, a calculated distance from the reference station to each satellite, and a satellite clock bias as well as an estimated receiver clock bias, and broadcasts them to the user application. The correction messages contain a Pseudo Range Correction (PRC) for DGPS, a Carrier Phase Correction (CPC) for CDGPS, and their time rates, Range Rate Correction (RRC) (Kee et al., 2008):

$$
\begin{gathered}
P R C=-(-b+I+T+\delta R)=d-\rho+\widehat{B} \\
C P C=-(-b-I+T+\delta R+N \lambda)=d-\varphi+\widehat{B}
\end{gathered}
$$

Where:

$\rho: \quad$ pseudo range measurement

$\varphi: \quad$ carrier phase measurement

$\lambda: \quad$ wavelength of the carrier phase

$N$ : integer ambiguity

$d$ : $\quad$ distance from the reference station to the satellite

$b$ : $\quad$ satellite clock bias error

$\widehat{B}$ : $\quad$ estimated clock bias of the receiver

I : $\quad$ ionospheric delay

T: $\quad$ tropospheric delay

$\delta R: \quad$ orbit error 
Unfortunately, the available accuracy for civilian applications is very poor because of all basic components in $(5,6)$. First of all, because of objective atmospheric delays (ionospheric and tropospheric). Although commercial DGPS (Differential GPS) service increases the accuracy up to several meters, it is still not sufficient for autonomous driving. Hence it is possible use an image processing to compensate this error. In spite of the fact that it is difficult to use image processing for recognition of a complex environment, it is possible to use it to determine some specified objects that haw obviously simple features. For example, lane tracking in highway, forward car tracking have been reported in (Vang et al., 2000). In this study, information about some specified objects is provided by a 3D map which is synthesized based on a real environment. The autonomous land vehicle uses these features to identify the indicated objects from the complex environments so as to position the vehicle's location. Because image processing consumes long time and positioning accuracy cannot satisfy the autonomous driving in many cases, environmental sensors, such as a 3D scanning laser rangefinder: ultrasonic environmental sensors, are also used.

Fig. 13 depicts the autonomous land vehicle used in the experiments (Vang et al., 2000). This vehicle was equipped with a color CCD camera, a GPS unit, a 3D scanning laser rangefinder, ultrasonic sensors, etc.

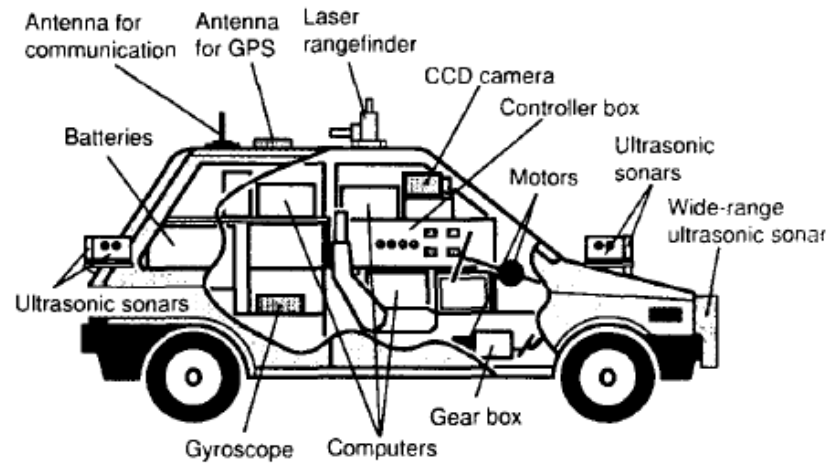

Fig. 13. The autonomous land vehicle used in the experiments (Vang et al., 2000)

Pedals and steering wheel are controlled with servomotors. The environmental sensing and control system is multitools. The position information from DGPS is used to retrieve environmental data from the $3 \mathrm{D}$ map. The $3 \mathrm{D}$ map provides the information about landmarks and features of some targets. Based the target features, the image processing system identify the landmarks by which the position of the vehicle is determined. An encoder attached to the wheel of a tire and a gyroscope are used for determination of the vehicle position and attitude. Because draft error may become unallowably large, these sensors are only for short distance measurement. A 3D scanning laser rangefinder is employed for compensation of the image processing system. The computers control the steering based on the information from the environmental recognition.

Positioning of GPS uses satellites. The resolution of the GPS used in the experiments is about $0.18 \mathrm{~m}$. However, the accuracy available for civilian applications is very poor. Even though DGPS makes the accuracy improved greatly, the positioning error is still insufficient for automatic driving. The positioning accuracy is not so good to navigate a vehicle, but enough to indicate the vehicle $\mathrm{t}$ o search some landmarks around the location. The synthesized 3D map provides geographic information that is referred by DGPS for positioning the vehicle's location. Because of the error of DGPS, some other information has 
to be given for the vehicle to compensate the positioning error of GPS. In study (Vang et al., 2000) the information about landmarks and their geometry and features is contained in the 3D map. The vehicle locates itself position by referring to the landmarks. The 3D map was synthesized by using OpenGL11. In the scene some objects are specified as landmarks for the image processing system to recognize as well to correct the positioning error caused by GPS. For the sake of simplicity of the image processing, some obvious features of the indicated objects are provided. The image processing identifies some objects indicated by the 3D map. By detecting the relative position to the indicated objects, the vehicle can determine itself position. Image processing is not always effective in any situation. In such case, a 3D scanning laser rangefinder is used. A laser rangefinder measures distance using time-of-flight method. It has advantages in positioning accuracy, detection speed in comparison with image processing. By horizontal and vertical scanning 2D or 3D maps can be obtained. The above discussed environmental sensors are effective for long range detection. To guarantee a safe driving the near surroundings of the vehicle have to be monitored. For this purpose, ultrasonic sensors were used. 8 ultrasonic sensors that have detection range of several meters are arranged around the vehicle. A no-scanning wide-range ultrasonic sensor was also developed and mounted in the front, of the vehicle. The experimental results of (Vang et al., 2000) demonstrated a higher performance in comparison with a video camera only.

But, if we'll analyze presented system, it can be classified more like combined system, than GPS-based as declared by authors. It shows one more again that robust and secure systems nowadays more and more became combined system, which shares strong skills of mentioned above classical approaches.

It is important to note, that, in our opinion, the GPS have a strong potential in a field of future machine vision design. Because in last ten years there is a lot of financial and intellectual investments to GPS and GPS-based technologies application. But this branch still can not be adopted as an independent base for machine vision, because of impossibility to scan nearby environment, to detect an arbitrary object in the scene. We are consider, that with years comes detailed GPS-maps with marked details in landscape. It will give a possibility to navigate a mobile object using dead reckoning principle (Ibeanusi et al., 1999), (Sadayuki Tsugawa, 1994). In this case a quantity of arbitrary objects in the scene will be minimized, and their location it is possible to fix by simple locator or UWB (Yu et al., 2008). But recently it is problematic use GPS as an independent vision tool. Another principle limitation of GPS is a nonlinear uncertainty growth for such dynamic application like mobile robot navigation for example.

Also it is essential to note that in present time any GPS application still have a fundamental problem of the method. The GPS technology cannot be used outside the radio signals receiving zone such as tunnels, mines, complex indoor spaces, up to that sometimes can not functioning correctly at dense cloudiness or overcast, etc.

A good example of "combined system" design is presented in (Petrov et al., p. 32, 1998). It is given the detailed consideration to the optical 3D scanner developed by company MetaCreations Real-Time Geometry Lab (www.metacreations.com/products/rtgeom). The principal design goals are portability, low cost, fast scanning speed, and photorealistic quality of models. To fulfill these requirements, a scanner is built mostly from off-the-shelf components. Also a set of rather complex software algorithms to help facilitate the scanning process is developed. The basic image processing algorithms were implemented in hardware. The scanning resolution is better than $1 \mathrm{~mm}$, while the acquisition time is sufficient. In the hardware design we avoided using complex optical schemes, instead transferring the complexity of the problem to algorithm development. This software- 
oriented approach allowed us to use simple components in an easy-to-manufacture design. Figure 14 shows a block diagram of Galatea scanner.

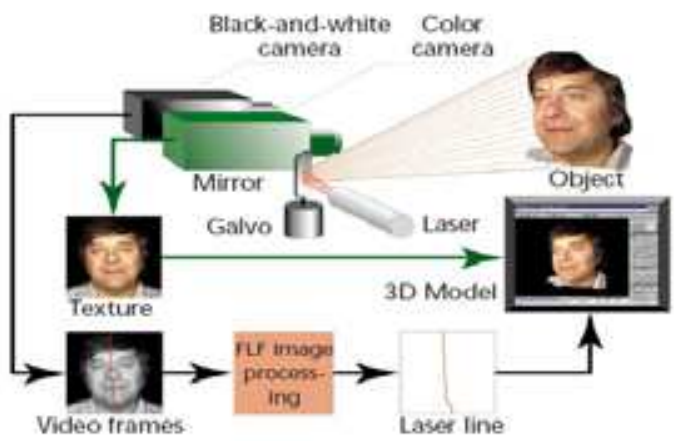

Fig. 14. Galatea scanner block-diagram. A galvanometric motor steers the laser stripe. The Fast Laser Finder (FLF) image processor processes the black-and-white camera's video stream. Merging the texture image of the color camera with the geometric model generates the final 3D model

The optical part consists of a laser-line generator, a galvanometric scanner, and black-andwhite and color cameras. It's quite natural to use a laser as the light source in a triangulation system. Diode lasers are compact and inexpensive, and can be easily focused down to their diffraction limit. The depth of field is considerably larger than the sensor camera's depth of field. For this reason, the laser does not require focusing during normal operation. In general, a properly designed scanner is eye safe, so that the system can be certified as a Class 1 laser according to the CDRH (Center for Devices and Radiological Health, Food and Drug Administration) classification. One of the major advantages of a laser source is that it can be easily filtered out from the ambient illumination using a narrow-bandpass filter. This allows laser-based digitizers to be operated in daylight conditions. Also, the laser signal can be easily filtered out from the color camera data, allowing the color texture and geometry to be captured simultaneously. The laser projection system consists of a laser-line generator and an ultrafast galvanometric scanner. The repeatability of average galvanometric scanners is about 20 microradians, while the nonlinearities are small enough to be calibrated for, if necessary. The positioning speed of such a motor exceeds 1,000 angles per second. An NTSC formatted high resolution black-and-white camera senses the geometry. A color CCD camera-installed between the laser projection system and the black-and-white cameracaptures the texture map. The scanning speed of 3D laser digitizers is limited by the rate of video cameras, typically 60 frames per second. Acquiring a 200-stripe resolution image would take about 3.3 seconds in a traditionally built digitizer. For many applications, including scanning the human body, this speed is not satisfactory. Using faster cameras provides a straightforward solution to increase speed. However, fast cameras tend to be rather expensive. To avoid using fast cameras, several solutions have been proposed.

Meanwhile, this system - as far as mentioned by authors collective - is designed as low cost solution for practical application where not necessary a high resolution. This example highlights the next fact. Any combined design always have larger coordinates error because a generalized system uncertainty is caused by complex cross-action of all components error sources and their interaction in this case is almost impossible to estimate by classic metrological methods. 


\section{Synthetic approach to machine vision system design}

The first principle conclusion from the methods overview is the next. The machine vision can be divided in a two general groups of methods:

- relatively fast, but rough form estimation methods of scene analysis (with significant uncertainty of form reconstruction);

- metrological methods based on the precise coordinates measurement. These methods requires for insignificant form reconstruction uncertainty a lot operating time and gives a enormous data set for memory storage. Practically it is almost impossible to use this group in real time for any dynamic system.

The second principle conclusion from the methods overview is the next. Competitive machine vision can not be based on any of these two bases. It must to share the main advantages of them: fast operability of the first one and precise 3D coordinates accuracy of the second one.

The third conclusion. Many modern scanners use "time of flight" to measure distance on the basis of the average speed of light in air. This measurement varies with air density, but because the speed of light is so great, these perturbations are very small for distances less than several kilometers. Other instruments use phase recognition to augment this technique for increased precision; however, this method is associated with an increase in data volume, which is undesirable.

The fourth conclusion from the methods overview: laser shows the high competitive ability over other scanning tools. It gives higher resolution, reasonable range and operation velocity. The weak point of laser technology is a limited power source of continuous scan time.

The key solution of this problem we are considering in a carefully thought over algorithm of information processing, very closely tied to each practical application. It must be flexibly used similar to human brain estimation: combine a very rough scene analysis in general, with furthering "zoom"-possibility for most problematic points surrounding (i.e. singular points). For this final fine stage it is only acceptable to provide a metrological accuracy method.

System optimized design according to open problem requirements will be explained on example of 3D laser Machine vision system, based on (Tyrsa et al., 2006, a), (Tyrsa et al., 2006, b), (Rivas et al., 2008).

\section{Multipurpose scanning vision system design}

It is considered in this paper a system with several characteristics previously referred; such as a high resolution, speed and versatility, can be used by our system in low cost.

In the most of fully autonomous navigation systems is used a vision system like this, our prototype can stand out among the other systems because has a very low cost; other advantage is the versatility, has a wide range of vision that can change to increase precision; and large sight depending of laser power.

This vision of environment is very similar to the human sight, has wide range of view, but can focus a specific area to get a high resolution and detailed image, so to make this task is not necessary modify hardware in prototype, just adjust some parameters in software 


\subsection{Original principle of 3D spatial coordinates measurement}

On fig.15, basic elements of machine vision system (MVS), placed as example on mobile robot (MR), are shown.
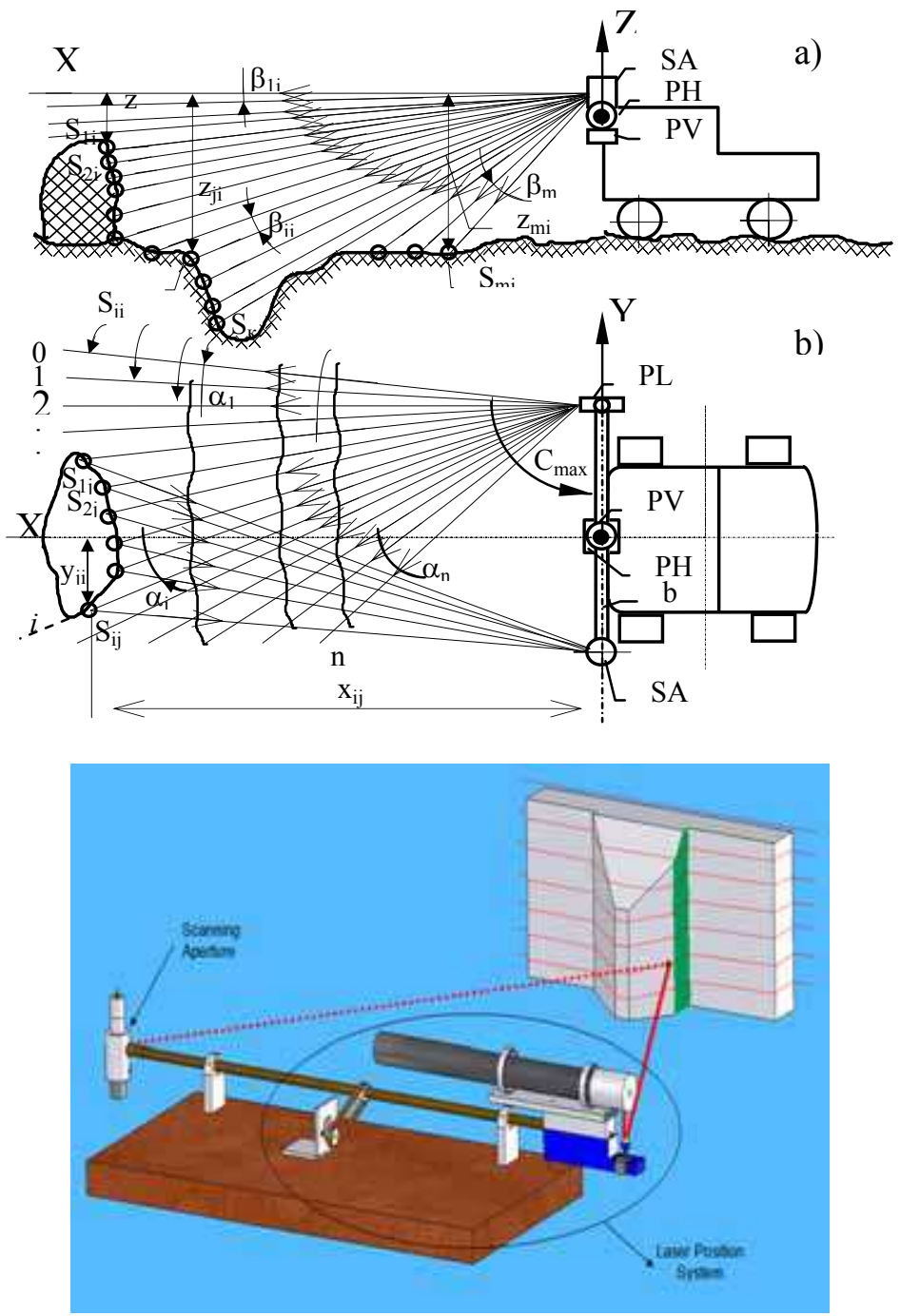

c)

Fig. 15. General principle of MVS functioning ( $\mathrm{a}$ - lateral view; $\mathrm{b}$ - top view; c - laser scanner prototype view)

MVS represents laser angle-distance-measuring system located in the upper forward part of MR. MVS contains a rather high-power laser with a collimator. The laser and the collimator are installed in own laser positioning system (PL) (fig.15, b). PL has its step drive, which on a command from the onboard computer can turn PL in a horizontal plane at each for one 
angle pitch. PL is on one end of a horizontal bar b. On the other end of the bar is located a scanning aperture (SA), shown separately on fig.16. The SA axle is perpendicular to a bar and plane XOY. The bar $\mathrm{b}$ is installed by its middle part in its own positioning system, containing horizontal (PH) and vertical (PV) step drives (fig.1).

MVS works in the next way (Tyrsa et al., 2006, a). By the command from the computer the bar is installed so that the SA rotation axis becomes perpendicular to plane XOY of MVS reference system (fig. 15). PL puts the laser with the collimator, for example, in an extreme right position. The axis of the collimator (with the help of PV-step drive) then takes extreme top position (above the horizon). The laser and the SA are switched on. SA is rotated by the electromotor EM. At each SA turn a laser ray should hit an obstacle, is reflected difusely by it (point Sij) and returns to mirror M (fig.16). At the moment when three objects: the point of reflection $\mathrm{Sij}$, the perpendicular to mirror $\mathrm{M}$ and the vertical axis of $\mathrm{SA}$ - takes their common plane, perpendicular to plane XOY while SA is rotating, an optical signal, having trevelled a path "Sij - mirror M - objective O - optical channel OC - photoreceiver PR ". It makes an electrical stop signal. A start signal is previously formed by SA by means of a zero-sensor (installed on a bar b axis).

Filling in a time interval $t_{B 1}=\mathrm{B}_{1} / \omega$, (where: $\mathrm{B}_{1}$ - angle between the bar axis (zero-sensor direction) and direction SA - Sij (fig.3); $\omega$ - SA rotation rate) with pulses of reference frequency $\mathrm{f}_{0}$, we shall receive a code $N_{B 1}=t_{B 1} \cdot f_{0}$. Rotation of SA cycle time $T_{2 \pi 1}=2 \pi / \omega$ is simultaneously filled with same impulses of reference frequency $\mathrm{f}_{0}$. The code $N_{2 \pi 1}=T_{2 \pi 1} f_{0}$ is formed. The angle $\mathrm{B}_{1}=2 \pi \cdot N_{B 1} / N_{2 \pi 1}$ is stored.

It is essentially to note, that this SA, or properly PSA (passive SA) as independent node have a very precise angle coordinates measurement accuracy. It can be used for navigation task as well as the static coordinates measurement (Tyrsa et al., 2004), for example for structural health monitoring of important civil engineering structures. Exactly, for inferior limit of stabilized reference oscillator of $1 \mathrm{MHz}$ and scanning velocity of 1 rev per sec, consequently we have an exact measurement scale of $1,000,000$ units for complete angle of $360^{\circ}$. Even such resolution $\left(0.00036^{\circ}\right.$ of angle) is an excellent for mentioned task. But it can be increased easy by reference frequency increase or scanner's velocity decrease.

When mirror SA has passed a direction towards point Sij, the laser is switched off to save power. The electric pulse goes to PL, the step-drive turns the laser with the collimator on an angle $\alpha_{1}$ along horizon (fig.15, b). By approach of SA mirror M to the MR 'view sector' the laser is switched on. If the laser ray in its new position hits an obstacle, a new point of reflection is formed and the measurement is re-cycled. If the laser ray does not hit an obstacle, no reflection will be, and a new point will not be formed. In this case, as well as in case when a point of reflection exists, the formation of a code $N_{62}$ begins with the signal of zero-sensor start pulse.

As soon as this code reaches some pre-determined value overlapping a range of possible code values corresponding to an operative sector, the counter, where codes NBi are formed, is installed on zero, and the laser is switched off. PL step-drive turns the laser on an angle $\alpha_{2}$. Cycles with measurements of angles on reflection points, if these are formed by obstacles, or single cycles of scanning, when the obstacle is not present, are repeated in the same order. Values of angles $\mathrm{Bi}$, in a cycle where reflection points took place, are stored. When the system PL stands in an extreme position $n$, stipulated by a minimum distance and 
a maximum angle of view, the bar positioning system step drive turns it round a horizontal axes by an angle $\beta_{1}$ (fig.15,a). The cycles with measurements of angles $B_{i}$ are recurred. Angles $\alpha_{i}$ are fulfilled in the back order from a position $n$ up to a position 0 .

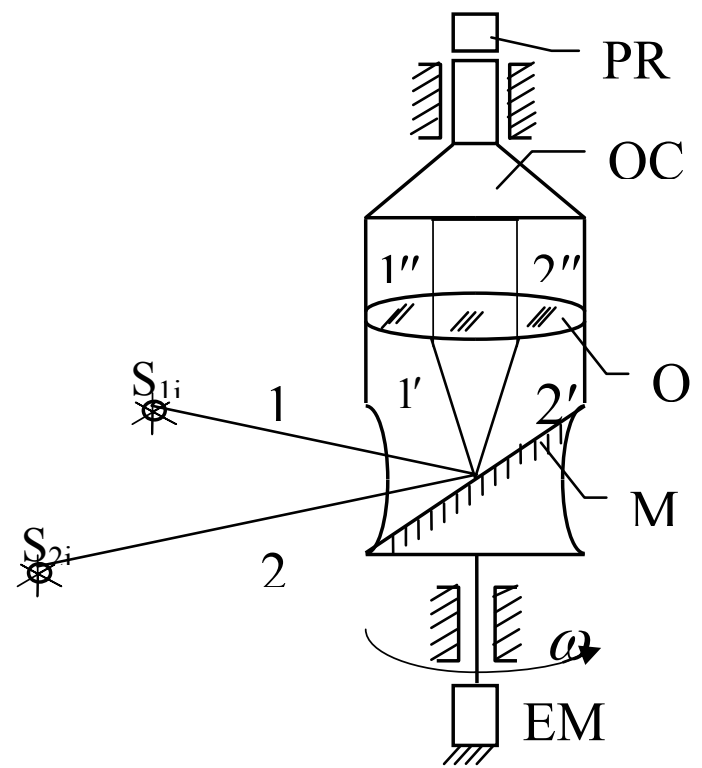

a)

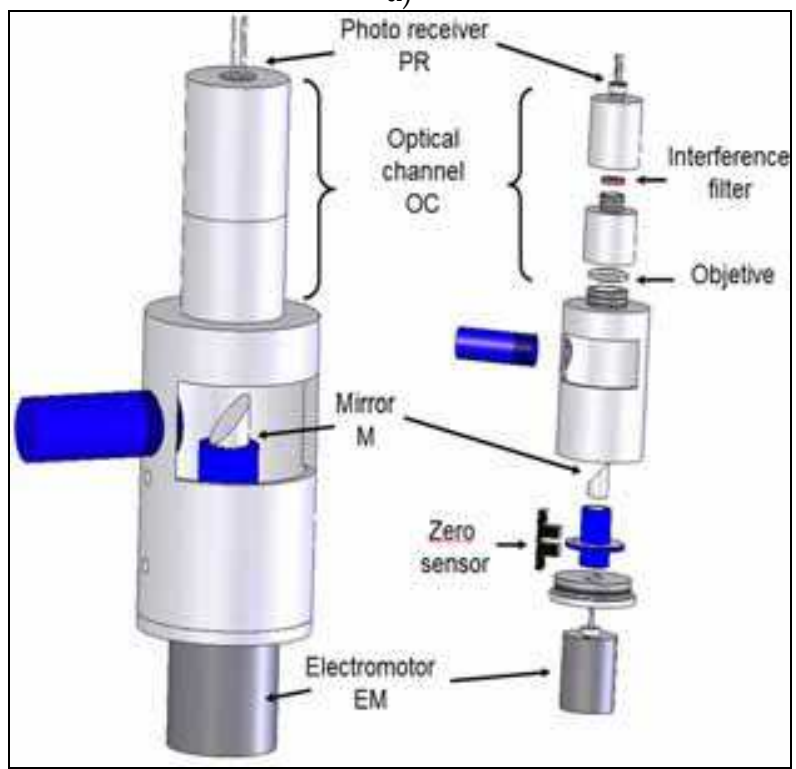

b)

Figure 16. Scanning aperture functioning principle (a), general design (b) 
After some position of a plane, where the laser ray moves in the plan from the right to the left or reverse, all fixed laser rays hit a surface under research, making points of reflection $\mathrm{S}_{\mathrm{ij}}$. If in this case laser emission power and distance to points of reflection as well as sensitivity of the photoreceiver are sufficient for formation of stop pulses, empty cycles of SA scanning will not exist. The measurement cycles are repeated until the plane, formed by a horizontal axes of a bar $b$ and the last point $S_{i j}$, deviates from a plane XOY by angle $\sum_{j=1}^{m} \beta_{j}$.

As mentioned above, data on angles $B_{i j}$ measured will be accumulated in computer memory. One of these angles is shown on Fig.3. A related angle $C_{i j}=C_{\max }-\sum_{i=1}^{i} \alpha_{i}$, where $C_{\max }-$ is an initial angle of PL position. Angles $C_{\mathrm{ij}}$, as well as angles $\sum_{j=1}^{j} \beta_{j}$, are fixed in memory simultaneously with the measured angles $\mathrm{B}_{\mathrm{ij}}$ during each cycle.

Using the theorem of sines as well as correlation between the sides and the height in a triangle represented on Fig.17.

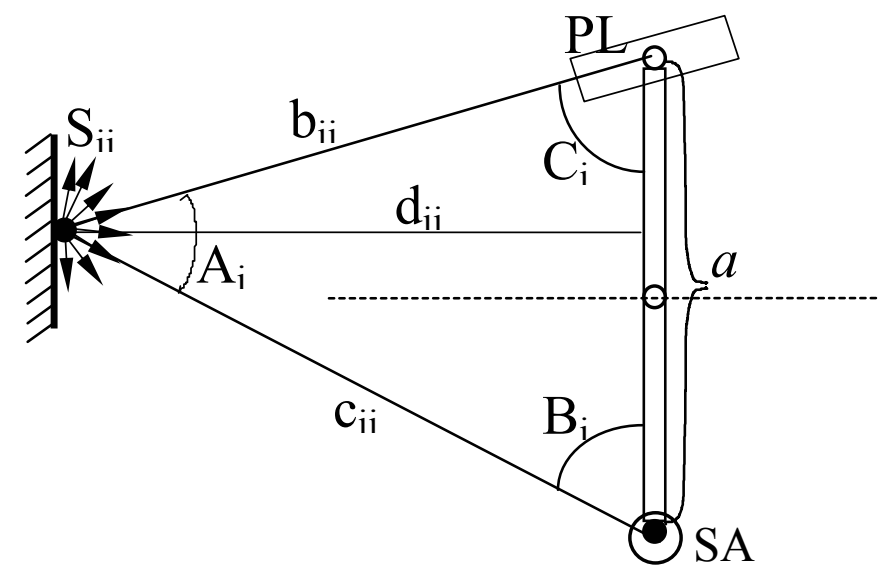

Fig. 17. Triangulation scheme for precise coordinates measurement

It is possible to find the formula for calculating sloping distances $\mathrm{d}_{\mathrm{ij}}$ from basis $b$ up to points highlighted by the laser

$$
\mathrm{d}_{\mathrm{ij}}=a \frac{\operatorname{Sin} B_{i j} \cdot \operatorname{Sin} C_{i j}}{\operatorname{Sin}\left[180^{\circ}-\left(B_{i j}+C_{i j}\right)\right]^{\prime}},
$$

where $a$ - is basic distance between rotation axes of PL and SA. It is accurately enough premeasured.

Using value of angles $\mathrm{B}_{\mathrm{ij}}, \mathrm{C}_{\mathrm{ij}}, \sum_{j=1}^{j} \beta_{j}$, and basis $a$, it is possible to calculate the Cartesian coordinates by each of the laser-highlighted points, in reference system OXYZ of MVS by the following formulas 


$$
\begin{gathered}
\mathrm{x}_{\mathrm{ij}}=a \cdot \frac{\operatorname{Sin} B_{i j} \cdot \operatorname{Sin} C_{i j} \operatorname{Cos} \sum_{j=1}^{j} \beta_{j}}{\operatorname{Sin}\left[180^{\circ}-\left(B_{i j}+C_{i j}\right)\right]}, \\
\mathrm{y}_{\mathrm{ij}}=a \cdot\left(\frac{1}{2}-\frac{\operatorname{Sin} B_{i j} \cdot \operatorname{Cos} C_{i j}}{\operatorname{Sin}\left[180^{\circ}-\left(B_{i j}+C_{i j}\right)\right]}\right) \text { at } \mathrm{B}_{\mathrm{ij}} \leq 90^{\circ}, \\
\mathrm{y}_{\mathrm{ij}}=-a \cdot\left(\frac{1}{2}+\frac{\operatorname{Sin} B_{i j} \cdot \operatorname{Cos} C_{i j}}{\operatorname{Sin}\left[180^{\circ}-\left(B_{i j}+C_{i j}\right)\right]}\right) \text { at } \mathrm{B}_{\mathrm{ij}}>90^{\circ}, \\
\mathrm{z}_{\mathrm{ij}}=a \cdot \frac{\operatorname{Sin} B_{i j} \cdot \operatorname{Sin} C_{i j} \operatorname{Sin} \sum_{j=1}^{j} \beta_{j}}{\operatorname{Sin}\left[180^{\circ}-\left(B_{i j}+C_{i j}\right)\right]} .
\end{gathered}
$$

Thus, the onboard computer with the help of TVS provides digital map-description of the terrain in a MR sector of view in an actual time scale. If in a direction of observation any obstacles shaped as a dredging or as a big item above a surface (in these places points Sij are heaped), MR, with the help of vertical step drive PV, turns TVS by an angle $\sum_{i=1}^{n} \alpha_{i}$ To the left or to the right depending on a position of obstacles in relation with axis OX, and "searches" a new sector.
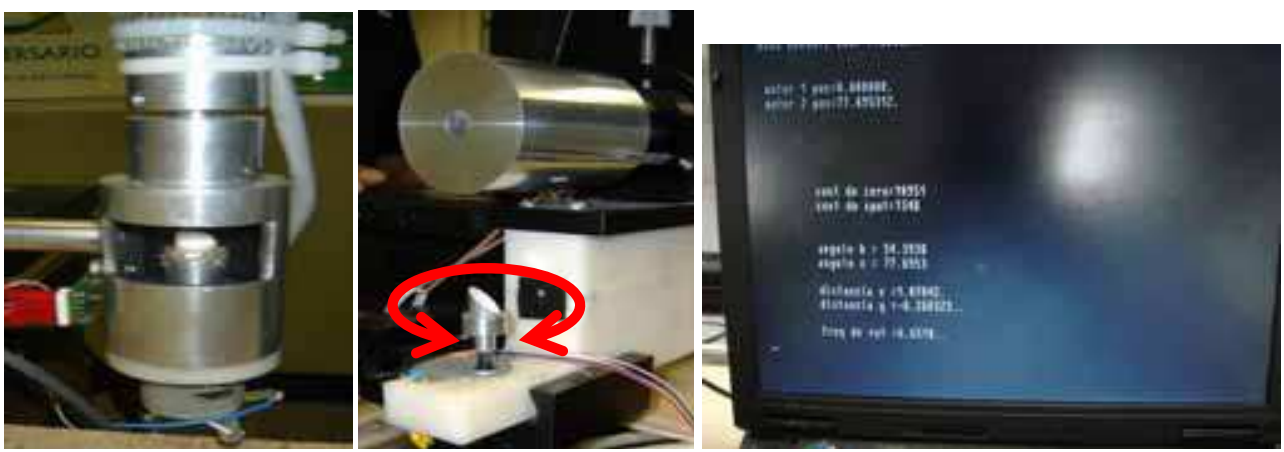

Fig. 18. Prototype parts (Scanning aperture; Emisor part (laser positioning system); and Software screen in function, taking epperimental data on obstacles

\subsection{Prototype construction and experimentations}

This prototype (Figs. 18 and 19) can be fabricated in very simple way using low-cost elements. A prototype was made with the follows elements: an aluminum base bar with $1 \mathrm{~m}$ of length, step motors to make vertical and horizontal scanning, source of light in this case a laser, a pair of mirrors with 45 degrees cut to redirection the light beam, in laser emisor part and scanning aperture (Fig.18), a photo receiver to convert light signal to electrical signal, a 
lens in the aperture part to get a fine convergence of light in photo receiver, a sensor (start) in the aperture part to detect and count revolutions of mirror2, and a interference filter to get only the selected source of light; this hardware is controlled by a laptop IBM Pentium II using "turbo c" program in MS-DOS where the measurement distances is stored (Fig.18), the connection to transfer data between hardware and software is by parallel port. The complete prototype (Rivas et al., 2008) is no longer than $1 \mathrm{~m}$, data can be stored in another system, and the prototype fabricated with all elements is shown in Fig.19 in action.

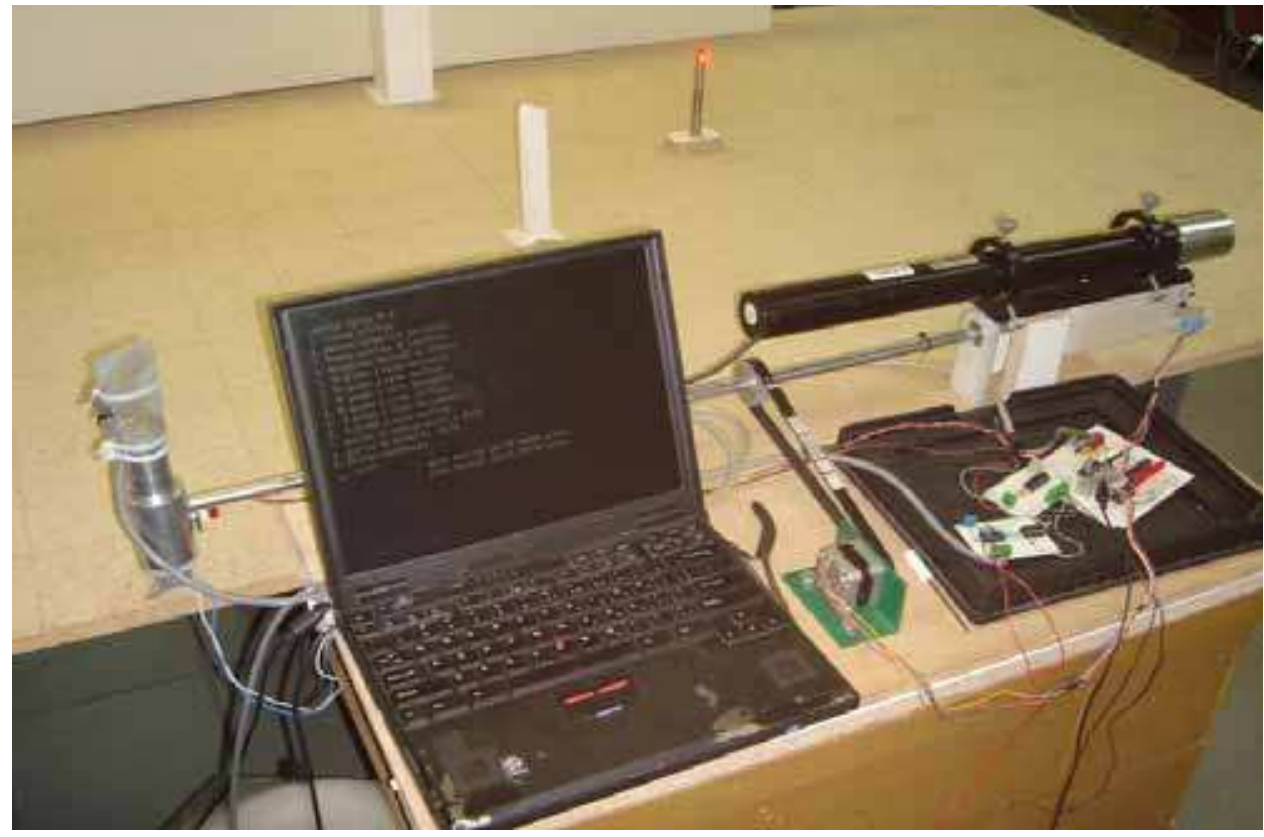

Fig. 19. Complete prototype system testing view.

This prototype was tested 100 times in different locations in grid nodes (see Figure 20) to verify accuracy on vision by different angles in $2 \mathrm{D}$, making tests on reflective and absorptive objects; in Table 2 are partially shown the measurement values by the prototype on a plane surface with grid-scale.

The values in the table 2 are shown graphically in (Figure 20). The violet points are distance values taken by the prototype working usual way; the yellow points are distances measured by the prototype but using an independent source of light in each point to measure (without prototype laser), finally the real distances can be shown by squares in the table base.

As it is evident, the range of error is very small in the center of the table, this center is the center of the prototype sight, and near both sides of the prototype sight angle the error is minimal. This prototype is very similar to human sight, at front the image view is very detailed and precise, both borders of angle of view have no much accuracy, this can be resolved rotating the vision of focus of the prototype exactly humans sight do.

Analyzing the measurement results (Fig.20) and its numerical values (Table 2), it is possible to say that all the experiments were carried out not less at $95 \%$ confidence level. And measurement uncertainty, especially its offset error, not overcomes a $5 \%$ threshold in any point, even in extended angle of sight where such accuracy is not obligatory. 


\begin{tabular}{|c|c|c|c|c|c|c|c|c|}
\hline \multirow{2}{*}{$\begin{array}{c}\text { Test } \\
\text { Point }\end{array}$} & \multicolumn{4}{|c|}{ Theoric Value } & \multicolumn{4}{|c|}{ Measured Value } \\
\hline & $\begin{array}{c}\mathrm{X} \\
(\mathrm{m})\end{array}$ & $\begin{array}{c}\mathrm{Y} \\
(\mathrm{m}) \\
\end{array}$ & $\mathrm{B}\left({ }^{\circ}\right)$ & $C\left(^{\circ}\right)$ & $x(m)$ & $y(m)$ & $B\left({ }^{\circ}\right)$ & $C\left({ }^{\circ}\right)$ \\
\hline B & 120 & 120 & 120,26 & 35,22 & 24,21 & 28,68 & 23,43 & 4,8 \\
\hline $\mathrm{D}$ & 100 & 100 & 116,57 & 33,69 & 99,83 & 104,48 & 118,46 & 32,87 \\
\hline $\mathrm{F}$ & 100 & 80 & 106,70 & 37,57 & 95,3 & 76,86 & 106,4 & 36,91 \\
\hline $\mathrm{H}$ & 120 & 60 & 94,76 & 47,49 & 122,92 & 63,49 & 96,38 & 47,28 \\
\hline $\mathrm{J}$ & 80 & 60 & 97,13 & 36,03 & 77 & 58,62 & 96,57 & 35,33 \\
\hline $\mathrm{L}$ & 80 & 40 & 82,87 & 41,63 & 79,99 & 42,15 & 84 & 40,95 \\
\hline $\mathrm{N}$ & 120 & 40 & 85,24 & 53,13 & 119,86 & 40,04 & 84,48 & 53,08 \\
\hline $\mathrm{O}$ & 120 & 20 & 75,96 & 59,74 & 119,22 & 19,48 & 76,01 & 59,76 \\
\hline $\mathrm{P}$ & 100 & 20 & 73,30 & 55,01 & 97,6 & 19,15 & 72,94 & 54,67 \\
\hline Q & 80 & 20 & 69,44 & 4 & 79,11 & 18,7 & 68,39 & 49,04 \\
\hline $\mathrm{R}$ & 60 & 20 & 63,43 & 40,60 & 60,33 & 21,7 & 64,3 & 40,07 \\
\hline$S$ & 40 & 20 & 53,13 & 29,74 & 39,14 & 19,59 & 52,07 & 29,35 \\
\hline $\mathrm{T}$ & 40 & 0 & 38,66 & 38,66 & 36,52 & 1,06 & 36,52 & 36,73 \\
\hline $\mathrm{U}$ & 0 & 0 & 0,11 & 50,19 & (1) & 0,1 & 49,39 & 50,625 \\
\hline V & 80 & 0 & 57,99 & 57,99 & 80,93 & 3,49 & 56,7 & 60,11 \\
\hline $\mathrm{W}$ & 100 & 0 & 63,43 & 63,43 & 103,58 & 1,51 & 63,45 & 64,68 \\
\hline$X$ & 120 & 0 & 67,38 & 67,38 & 120,72 & $-4,7$ & 66,23 & 69,43 \\
\hline$Y$ & 120 & -20 & 59,74 & 75,96 & 118,54 & $-18,36$ & 59,95 & 75,05 \\
\hline $\mathrm{Z}$ & 100 & -20 & 55,01 & 73,30 & 96,72 & $-18,37$ & 54,7 & 71,9 \\
\hline A1 & 80 & -20 & 48,81 & 69,44 & 75,79 & $-18,33$ & 48,03 & 67,32 \\
\hline B1 & 60 & -20 & 40,60 & 63,43 & 58 & $-18,53$ & 39,5 & 61,52 \\
\hline $\mathrm{C} 1$ & 40 & -20 & 29,74 & 53,13 & 36,17 & $-20,31$ & 27,3 & 50,62 \\
\hline D1 & 60 & -40 & 33,69 & 80,54 & 58,05 & $-38,63$ & 33,6 & 78,92 \\
\hline E1 & 80 & \begin{tabular}{|l|}
-40 \\
\end{tabular} & 41,63 & 82,87 & 77,46 & $-38,26$ & 40,46 & 81,38 \\
\hline $\mathrm{F} 1$ & 100 & -40 & 48,01 & 84,29 & 97,1 & $-38,02$ & 46,94 & 82,96 \\
\hline H1 & 120 & -60 & 47,49 & 94,76 & 123,68 & $-61,03$ & 48,21 & 95,09 \\
\hline $\mathrm{J} 1$ & 80 & -60 & 36,03 & 97,13 & 78,9 & $-59,73$ & 35,37 & 97,03 \\
\hline L1 & 80 & -80 & 31,61 & 110,56 & 80,52 & $-80,21$ & 31,81 & 110,56 \\
\hline N1 & 120 & -80 & 42,71 & 104,04 & 121,01 & $-78,73$ & 43,34 & 103,35 \\
\hline P1 & 100 & $\begin{array}{c}- \\
100 \\
\end{array}$ & 33,69 & 116,57 & 101,65 & $-96,18$ & 35,06 & 114,43 \\
\hline R1 & 100 & $\begin{array}{c}- \\
120\end{array}$ & 30,47 & 124,99 & 111,73 & 126,15 & 2,09 & 124,27 \\
\hline
\end{tabular}

Table 2. Experimental results of point-coordinates measurement (Rivas et al., 2008) 


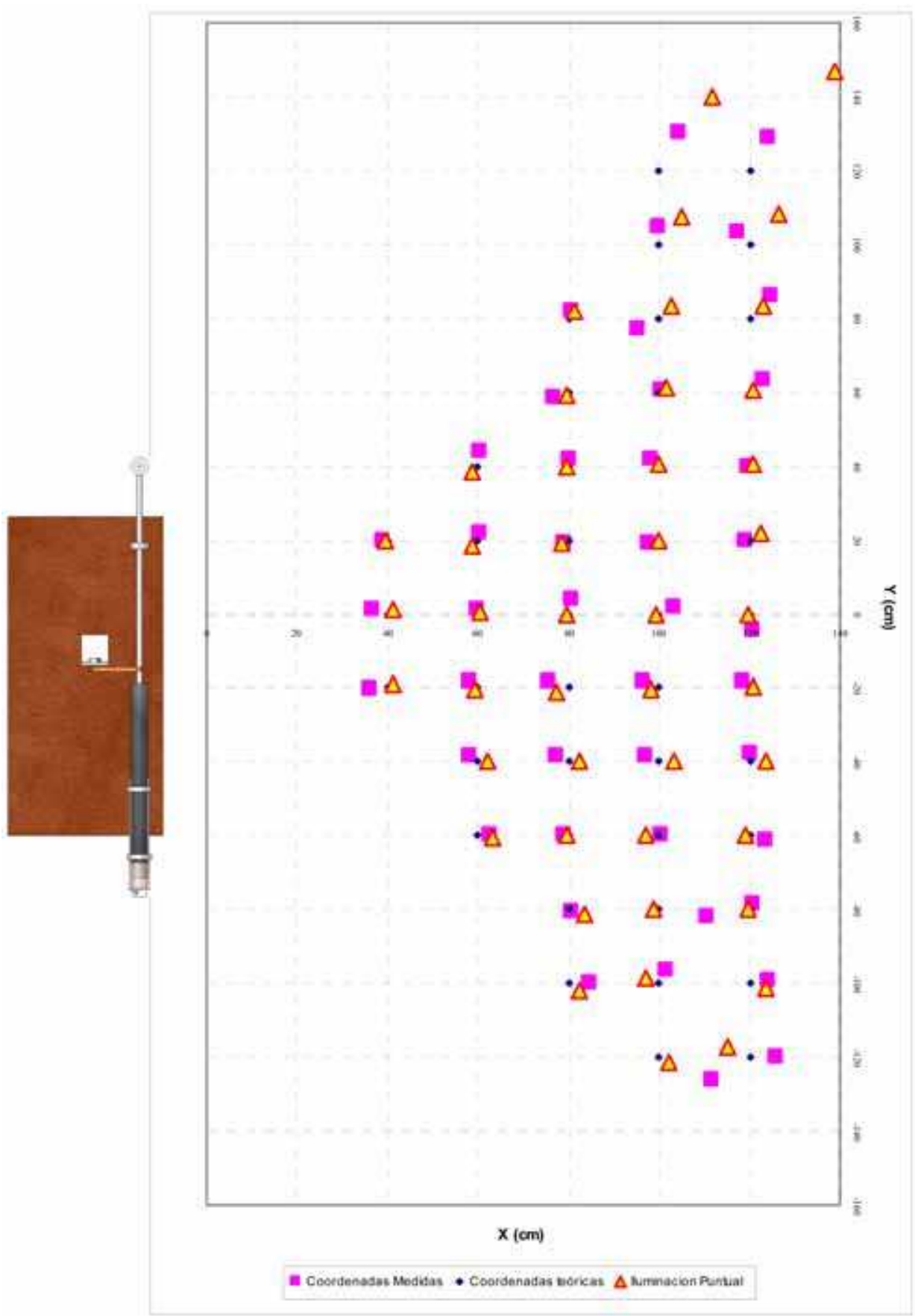

Fig. 20. Experimental graphic of 2D points measurement and obstacle positioning 
Also detailed analyzis of scanning aperture original construction (Fig. 16, 18) and principle deduce us to conclusion that it is possible to obtain measurement results with the same uncertainty from highlighted points of the different attitudes inside precertain angle of view. It is significant advantage of the presented device over other known laser triangulation scanners (Forman \& Parry, 2001), (Pierce et al., 1992), (Surmann et al., 2003). In general behavior of uncertainty repeats graphic presented on Figure 6 in (Pierce et al., p. $1765,1992)$, however accuracy nearby edge in our system is more advanced. Also accuracy (coordinate measurement offset) in a central part of scanner's angle of view is 2-7 times better in a different checked points respect to (Pierce et al., 1992) for example.

\subsection{Prototype application for static monitoring}

The above-described passive scanning aperture (Figs. 16 and 18) (PSA) independently can be able for static object deformation monitoring (Tyrsa et al., 2004). PSA, or POS (passive optical scanner), using the vertical axis of rotation is destined for the measurement of the horizontal angles. Let's call it a vertical POS. The vertical angles between the "emissive beacons"(EBs) are measured by the POS with the horizontal axis of the rotation. This POS is the horizontal POS. The system, which consists of the vertical and horizontal POSs, permits to measure the displacement of the EB in horizontal and vertical planes.

Fig. 2 shows the adoption of the horizontal POS for the realization of the bridge points monitoring in a vertical plane.

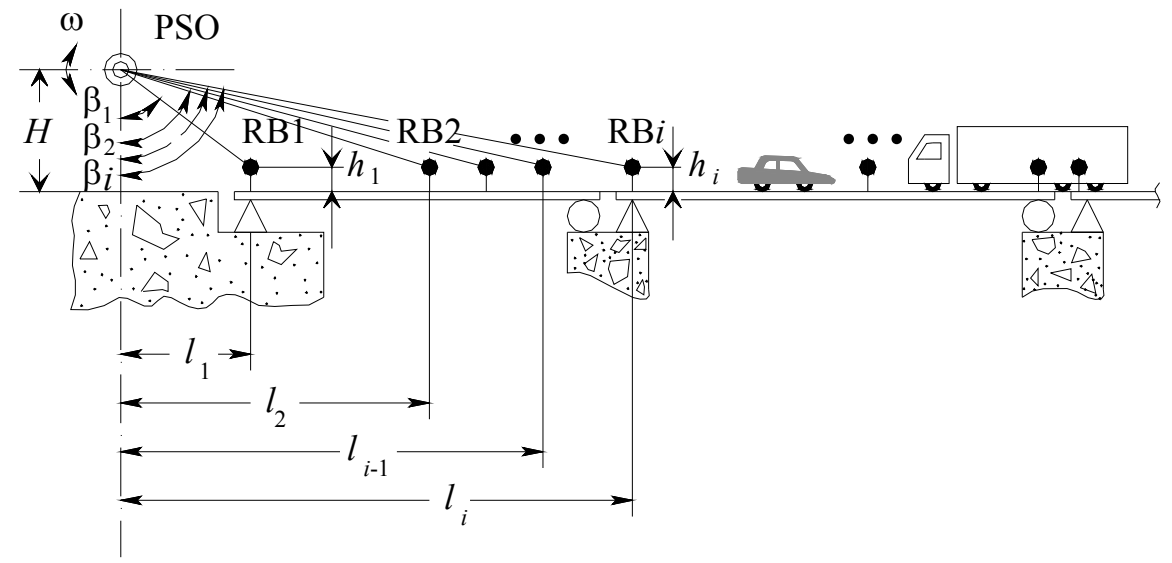

Fig. 21. Horizontal POS placement for bridge monitoring

The POS is placed outside the bridge and measures the vertical angles $\beta_{i}$. The EBs are installed with the same height $h_{i}$ above the bridge surface. As EB it is possible to use any sufficiently cheap light source, for example mentioned above lamp HRQ3010-5071. The distances $l_{i}$ are measured during the installation of the EB.

In the absence of a deformation, for each $\mathrm{EB}_{\mathrm{i}}$, the following expression is valid:

$$
\frac{H-h}{l_{i}}=\tan \left(\frac{\pi}{2}-\beta_{i}\right)
$$


For the bridge deformation with the magnitude $\Delta h_{i}$ in the placement point of the beacon $\mathrm{EB}_{\mathrm{i}}$, the angle $\beta_{i}$, measured by the POS, will change on the magnitude $\Delta \beta_{i}$. As a result the expression (13) will receive the form:

$$
\frac{H-h \pm \Delta h}{l_{i}}=\tan \left(\frac{\pi}{2}-\beta_{i} \pm \Delta \beta_{i}\right)
$$

Therefore:

$$
\Delta h_{i}=(H-h)-l_{i} \tan \left(\frac{\pi}{2}-\beta_{i} \pm \Delta \beta_{i}\right) .
$$

In a similar manner the POS for tunnel monitoring is installed. In extended tunnels several functionally connected POS can be placed along its axis.

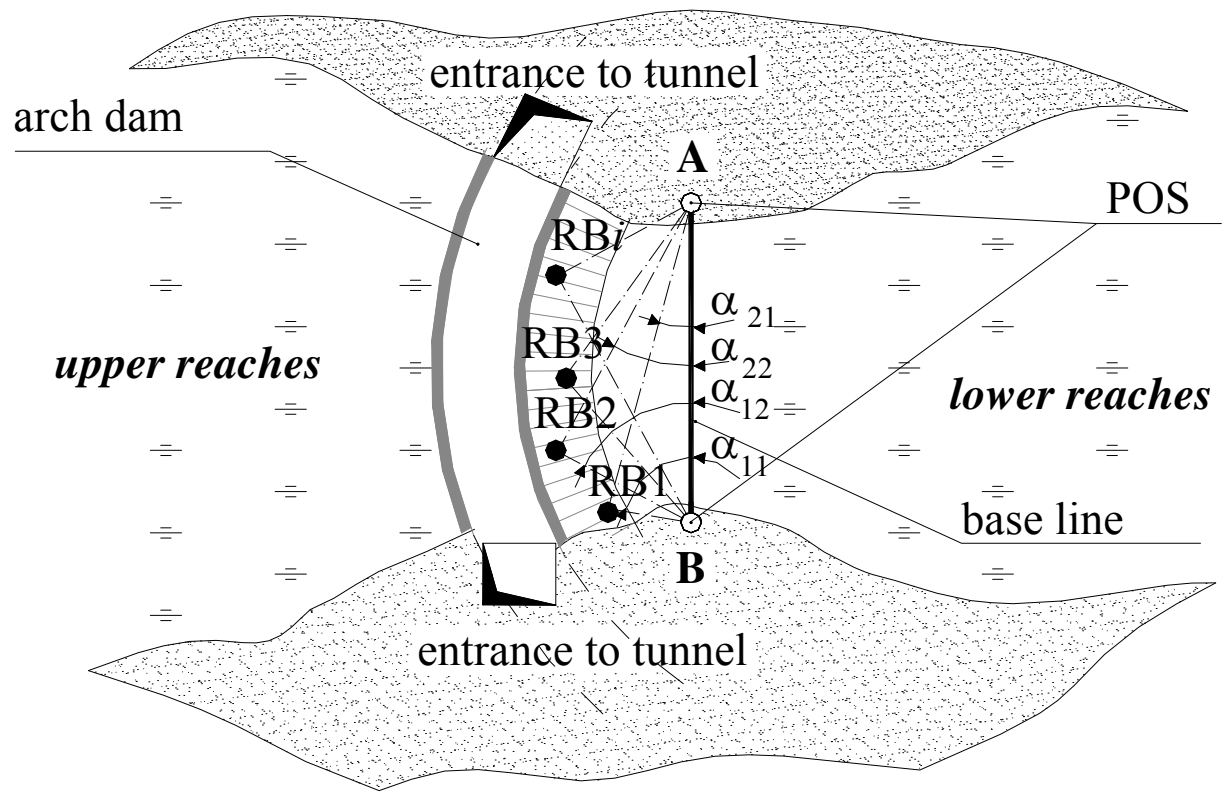

Fig. 22. Placement of two vertical POSs for arch dam monitoring.

Fig. 22 shows the placement of two vertical POSs for the arch dam monitoring of a hydroelectric power station. The POSs measure in pairs the horizontal angles $\alpha_{1 i}$ and $\alpha_{2 i}$ between the base line $A B$ and the $\mathrm{EB}_{\mathrm{i}}$. The length of the base line $D$ and the Cartesian coordinates of the POS are determined beforehand by known geodetic methods. The angles $\angle \mathrm{EB}_{\mathrm{i}}$ are found by the formula:

$$
\angle \mathrm{RB}_{\mathrm{i}}=180^{\circ}-\left(\alpha_{1 i}+\alpha_{2 i}\right)
$$


According the sine theorem we find the triangles sides, which have the vertex $A, B, E_{i}$; and the coordinates $x_{i}, y_{i}$ of the points $\mathrm{EB}_{\mathrm{i}}$, in the national system or conditional Cartesian coordinate system. The change of coordinates of the points $R_{i}$ in the repeated measurements of the angles $\alpha_{1 i}$ and $\alpha_{2 i}$ reveal the dam deformation.

\section{Conclusions}

Presented research was provided with a general goal to design simple and secure method for 3D laser scanning of any unknown surface placed on a variable distance from scanner location. Other advantage of a present system over other laser triangulation system is a higher in principle order of exactitude in a part of 3D coordinates calculation. Preliminary prototype testing is shown

- This theoretical principle can be realized with modern electronic and electromechanic devices in low cost;

- With completely acceptable operation velocity this device can detect an obstacle and to cover it with digital 3D map of $n$ points on it's surface;

- This factor $n$ is easy possible to change as only one variable in software; such way provide basic principle "more points - less uncertainty, but more operating time". This task must be solved carefully for each practical application;

- Coordinates calculation uncertainty is less than any known laser triangulation with electronic scanning because of exact surveying techniques principle using;

- This system in a closest way simulates the human binocular vision; as shown, the best exactitude can be achieved in a center of angle of view, and nearby both edges the picture is not has so sharpness. It possible to overcome by base bar rotation;

- These techniques can be implemented with insignificant changes for various practical applications, such as large engineering structures Structural Health Monitoring, mobile robot navigation, microsurface inspections, etc.

\section{References}

Allen, D.; Park, G.; \& Farrar, C. (2005). Overview of Wireless Active Sensing Systems for Structural Health Monitoring, In Structural health monitoring, Fu-Kuo Chang, page numbers (1578-1585), DEStech Publications, ISBN: 1-932078-51-7

Athavale, N.; Ragade, R.; Cassaro M.; \& Fenske, T. (1990). A prototype for KYBAS: the Kentucky Bridge Analysis System. Proceedings of the 3rd international conference on Industrial and engineering applications of artificial intelligence and expert systems, pp. 781-789, Vol. 2, 1990

Baltsavias, E. P. (1999), a. A comparison between photogrammetry and laser scanning. ISPRS Journal of Photogrammetry \& Remote Sensing, Elsevier, Vol. 54, (July 1999) page numbers (83-94)

Baltsavias, E. P. (1999), b. Airborne laser scanning: basic relations and formulas. ISPRS Journal of Photogrammetry \& Remote Sensing, Elsevier, Vol. 54, (July 1999) page numbers (199-214)

Bellian, J. A.; Kerans, C. \& Jennettem, D. C. (2005). Digital outcrop models: applications of terrestrial scanning lidar technology in stratigraphic modeling. Journal of 
sedimentary research, SEPM , Vol. 75, No. 2, pp. 166-176, ISBN 1527-1404/05/075166, march, 2005

Benedetti, M.; Donelli, M.; Massa, A. \& Rosani. A. (2004). An innovative microwave imaging technique for non destructive evaluation: applications to civil structures monitoring and biological bodies inspection. IEEE Proceedings of International "Imaging Systems and Techniques, (IST-2004)",(may 2004) page numbers (91 - 94)

Chaumette, F. \& Hutchinson, S. (2006). Visual servo control PART I: basic approaches. IEEE Robotics \& Automation Magazine, December 2006, pp.82-90.

Chellappa, R.; Roy-Chowdhury, A. K. \& Zhou, Sh. K. (2005). Recognition of humans and their activities using video. Morgan \& Claypool Publishers, ISBN 1598290061

Diosi, A. \& Kleeman, L. (2005). Laser scan matching in polar coordinates with application to SLAM. Proceedings of IEEE/RSJ International Conference on Intelligent Robots and Systems (IROS 2005), pp. 3317- 3322, ISBN: 0-7803-8912-3, august 2005

França, J.; Gazziro, M.; Ide, A. \& Saito, J. (2005). A 3D scanning system based on laser triangulation an variable field of view, Proceedings of the 2005 International Conference on Image Processing (ICIP 2005), pp. 425-428, IEEE Volume 1, Italy, 2005, Genoa

Forman, P. \& Parry, I. (2001). Rapid Data Collection at Major Incident Scenes using Three Dimensional Laser Scanning Techniques. 2001 IEEE 35th International Carnahan Conference on Security Technology, pp. 60-67, october 2001

Hada, Y. \& Takase, K. (2001). Multiple Mobile Robot Navigation Using The Indoor Global Positioning System (iGPS). Proceedings of IEEE/RSJ International Conference on Intelligent Robots and Systems, Vol. 2, pp. 1005-1010, ISBN: 0-7803-6612-3, USA, Maui, HI, 10/29/2001 - 11/03/2001

Hancock, J. ; Langer, D.; Hebert, M.; Sullivan, R.; Ingimarson, D.; Hoffman, E.; Mettenleiter, M. \& Fredlich, C. (1998). Active Laser Radar For High-Performance Measurements, IEEE International Conference on Robotics \& Automation, pp 1465-1470, Belgium, Leuven, 1998

Handbook of Optical and Laser Scanning. (2004).Edited by Gerald F. Marshall, 769 p., ISBN: 08247-5569-3, Marcel Dekker, Inc., New York - Basel, 2004

Hosmer, P. (2004). Use of Laser Scanning Technology for Perimeter Protection. IEEE Aerospace and Electronic Systems Magazine, vol. 19, page numbers (13-17), 2004

Ibeanusi, U.; Lafond-Favieres, V. ; McLawhorn, L. \& Anderson, S. (1999). Understanding robot navigation and control, Proceedings of the 37th annual ACM Southeast Regional Conference, 7p, CD version, 1999

Itti, L. \& Baldi, P. (2005). A principled approach to detecting surprising events in video. Proceedings of IEEE CVPR2005, pp. 631- 637, vol. 1, International Conference on Computer Vision and Pattern Recognition, 20-25 June 2005

Kee, C.; Park, B.; Kim, J.; Cleveland, A.; Parsons, M. \& Wolfe, D. (2008). A Guideline to Establish DGPS Reference Station Requirements. The journal of navigation. Royal Institute of Navigation-Cambridge Journals (2008), 61, page numbers (99-114)

Lavelle, J. P. ; Schuet, S. R. \& Schuet, D. J. (2004). High Speed 3D Scanner with Real-Time 3D Processing. Proceedings of ISA/IEEE Sensors for Industry Conference, pp.102- 108, ISBN: 0-7803-8143-2, 2004

Lichti, D. D. ; Stewart, M. P.; Tsakiri, M. \& Snow, A. J. (2000). Benchmark tests on a ThreeDimensional Laser Scanning system. Department of Spatial Sciences of Curtin University of Technology, Geomatics Research Australasia, 23 p., 2000 
Liu, F.; Bhakar, S.; Fevens, T. \& Mudur, S. (2006). Environment Lighting for Point Sampled Geometry. Proceedings of International Conference on Computer Graphics, Imaging and Visualisation (CGIV'06), pp. 522-527, 2006

Liwen Dai, Jinling Wang, Rizos, C. \& Shaowei Han. (2002). Pseudo-satellite applications in deformation monitoring. GPS Solutions Journal, Springer Berlin / Heidelberg, Vol. 5, No 3, (January, 2002), page numbers (80-87), ISSN: 1080-5370 (Print) 1521-1886 (Online)

Mallet, L.; Lee, B.; Staszewski, W. \& Scarpa, F. (2004). Structural health monitoring using scanning laser vibrometry: II. Lamb waves for damage detection. IOP electronic journals; Smart Mater. \& Struct., 13, (2004), page numbers (261-269)

Mordohai, P. \& Medioni, G. (2006). Tensor Voting: A Perceptual Organization Approach to Computer Vision and Machine Learning, Morgan \& Claypool Publishers, ISBN: 1598291009

Noll, R. \& Krauhausen, M. (2003). Multibeam laser triangulation for the measurement of geometric features of moving objects in production lines. Conference on Lasers and Electro-Optics Europe CLEO/Europe, p. 468, ISBN: 0-7803-7734-6, 22-27 June 2003

Ohno, H.; Naruse, H.; Kurashima, T.; Nobiki, A.; Uchiyama, Y. \& Kusakabe, Y. (2002). Application of Brillouin Scattering-Based Distributed Optical Fiber Strain Sensor to Actual Concrete Piles. IEICE TRANSACTIONS on Electronics, Vol. E85-C, No.4, (2002), page numbers (945-951)

Peng-Hsiang Weng \& Fu-Jen Kao. (2004). Modulation Based Time Resolved Laser Scanning Microscopy. The Second Asian and Pacific Rim Symposium on Biophotonics, APBP 2004, pp.97- 98, ISBN: 0-7803-8676-0, december 2004

Petrov, M.; Talapov, A.; Robertson, T. Lebedev, A.; Zhilyaev, A. \& Polonskiy, L. (1998). Optical 3D Digitizers: Bringing Life to the Virtual World. IEEE Computer Graphics and Applications, Vol., 18, (May/Jun 1998), page numbers (28-37). Issue: 3, ISSN: 0272-1716

Pierce, D.S.; Ng, T.S. \& Morrison, B.R. (1992). A Novel Laser Triangulation Technique for High Precision Distance Measurement, Proceeding of IEEE Industry Aplications Society Annual Meeting, vol.2, pp. 1762-1769, USA, october, 1992, Houston

Retscher, G. (2007). Test and Integration of Location Sensors for a Multi-sensor Personal Navigator. The Journal of Navigation, 60 (1), page numbers (107-117)

Rivas, M.; Sergiyenko, O.; Aguirre, M.; Devia, L.; Tyrsa, V. \& Rendón, I. (2008). Spatial data acquisition by laser scanning for robot or SHM task. Proceeding of IEEE/IES International Conference ISIE-2008 pp.1458-1463, ISBN 978-1-4244-1666-0, UK, Cambridge, 30June - 2 July, 2008

Riza N. A. \& Muzammil, A. A. (2003). Code-multiplexed optical scanner, In: Applied Optics, vol. 42, pp. 1493-1501, 2003

Sadayuki Tsugawa. (1994). Vision-Based Vehicles in Japan: Machine Vision Systems and Driving Control Systems. IEEE Transactions on industrial electronics, Vol. 41, No. 4, pp.398-405, august, 1994

Sahba, K.; Alameh, K. E. \& Smith, C. (2006). A Proposed Motionless Laser Scanning Architecture for Perimeter Security. Proceedings of 40th ICCST2006, pp. 9-16, ISBN: 1-4244-0174-7, Lexington, KY, October 17, 2006

Slob, S. \& Hack., R. (2004). 3D Terrestrial Laser Scanning as a New Field Measurement and Monitoring Technique, In: Engineering Geology for Infrastructure Planning in Europe, Springer Berlin, page numbers (179-189), Heidelberg 
Selectes papers on CCD and CMOS imadges. (2003). SPIE Press Bellingham, editor Moon Gi Kang, General editor Brian J.Thompson,Vol. MS177, 636p., ISBN 0-8194-5114-2 USA, Washington, 2003

Soini, A. (2001). Machine vision technology take-up in industrial application. Proceedings of the 2nd International Symposium on Image and Signal Processing and Analysis ISPA, pp. 332-338, ISBN: 953-96769-4-0, Pula, Croatia

Steinle, E. \& Vogtle, T. (2000). Effects of different laser scanning modes on the results of building recognition and reconstruction. International Archives of Photogrammetry and Remote Sensing, 2000 - www-ipf.bau-verm.uni-karlsruhe.de. 8p.

Stewart, M. P. \& Tsakiri, M. (2002). The Application of GPS to Dam Surface Monitoring. Journal of Geospatial Engineering, Vol.3, No.1, page numbers (45-57)

Stutz, G. (2005). Guiding Light, SPIE's magazine, vol. 5, page nnumbers (25-27), 2005

Surmann, H.; Lingemann, K.; Nuchter, A. \& Hertzberg, J. (2003). A 3D laser range finder for autonomous mobile robots. Robotics and Autonomous Systems, Issues 3-4, vol. 45, pp. 181-198, december 2003

Tao Dai; Xiang Chen; Drake, R. \& Jun Yang. (2005). Vision Sensor Based Tracking and Focusing of 3-D Singular Points. Proceedings of 2005 IEEE International Conference on Mechatronics and Automation, Vol. 3, pp. 1312-1317, ISBN: 0-7803-9044-X, Canada, 2005, Niagara Falls, Ont.

Tunstel, E. (1995). Fuzzy spatial map representation for mobile robot navigation. Proceedings of the 1995 ACM symposium on Applied computing, pp. 586 - 589, ISBN:0-89791-6581, USA, (1995), Nashville, Tennessee

Tyrsa, V.E.; Burtseva, L.; Rivas Lopez, M.; \& Tyrsa, V.V. (2004) Monitoring of civil engineering structures. Proc. of SPIE Int. Conf. Health monitoring and smart nondestructive evaluation of structural and biological systems III, 5394, pp. 485-492, USA, 2004, San-Diego

Tyrsa, V. Ye.; Sergiyenko, O.; Burtseva, L.; Bravo-Zanoguera, M.; Devia, L.; Rendon, I. \& Tyrsa, V. V. (2006), a. Mobile Transport object control by technical vision means. Proceeding of IEEE International Conference CERMA2006, Vol. II, p.74-79, ISBN: 07695-2569-5/ ISBN13 978-0-7695-2569-3, Mexico, Cuernavaca, September 2006

Tyrsa, V.Ye.; Sergiyenko, O.Yu.; Tyrsa, V.V.; Bravo, M.; Devia, L. \& Rendon, I. (2006), b. Mobile Robot navigation by Laser Scanning Means. Proceeding of $3^{\text {rd }}$ International "Conference on Cybernetics and Information Technologies, Systems and Applications CITSA - 2006", Vol. I, p. 340-345, ISBN: 980-6560-82-5 (collection)/ 980-6560-83-3 (volume I), USA, July 20-23, 2006, Orlando, Florida

Vang, L.; Emura, T. \& Ushiwata, T. (2000). Automatic Guidance of a Vehicle Based on DGPS and a 3D Map, Proceedings of IEEE Intelligent Transportation Systems, pp.131-136, ISBN: 0-7803-5971-2, USA, october 2000, Dearborn, MI

Wehr, A. \& Lohr, U. (1999). Airborne laser scanning - an introduction and overview. ISPRS Journal of Photogrammetry and Remote Sensing, Elsevier, Vol. 54, Issues 2-3, (July 1999) page numbers (68-82) et al.,

Winkelbach, S.; Molkenstruck, S. \& Wahl, F. (2006). Low-cost Laser Range Scanner and Fast Surface Registration Approach. Proceedings of DAGM 2006, Springer Berlin Heidelberg, (2006) page numbers (718-728)

Yu, H.; Aguado, E.; Brodin, G.; Cooper, J. ; Walsh D. \& Strangeways H. (2008). UWB Positioning System Design: Selection of Modulation and Multiple Access Schemes. The Journal of Navigation, 61, page numbers (45-62) 


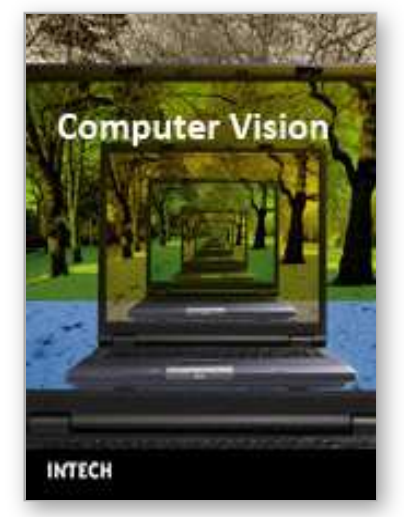

\author{
Computer Vision \\ Edited by Xiong Zhihui
}

ISBN 978-953-7619-21-3

Hard cover, 538 pages

Publisher InTech

Published online 01, November, 2008

Published in print edition November, 2008

This book presents research trends on computer vision, especially on application of robotics, and on advanced approachs for computer vision (such as omnidirectional vision). Among them, research on RFID technology integrating stereo vision to localize an indoor mobile robot is included in this book. Besides, this book includes many research on omnidirectional vision, and the combination of omnidirectional vision with robotics. This book features representative work on the computer vision, and it puts more focus on robotics vision and omnidirectioal vision. The intended audience is anyone who wishes to become familiar with the latest research work on computer vision, especially its applications on robots. The contents of this book allow the reader to know more technical aspects and applications of computer vision. Researchers and instructors will benefit from this book.

\title{
How to reference
}

In order to correctly reference this scholarly work, feel free to copy and paste the following:

Moises Rivas Lopez, Oleg Sergiyenko and Vera Tyrsa (2008). Machine Vision: Approaches and Limitations, Computer Vision, Xiong Zhihui (Ed.), ISBN: 978-953-7619-21-3, InTech, Available from: http://www.intechopen.com/books/computer_vision/machine_vision_approaches_and_limitations

\section{INTECH}

open science | open minds

\section{InTech Europe}

University Campus STeP Ri Slavka Krautzeka 83/A 51000 Rijeka, Croatia Phone: +385 (51) 770447 Fax: +385 (51) 686166 www.intechopen.com

\author{
InTech China \\ Unit 405, Office Block, Hotel Equatorial Shanghai \\ No.65, Yan An Road (West), Shanghai, 200040, China \\ 中国上海市延安西路65号上海国际贵都大饭店办公楼405单元 \\ Phone: +86-21-62489820 \\ Fax: +86-21-62489821
}


(C) 2008 The Author(s). Licensee IntechOpen. This chapter is distributed under the terms of the Creative Commons Attribution-NonCommercialShareAlike-3.0 License, which permits use, distribution and reproduction for non-commercial purposes, provided the original is properly cited and derivative works building on this content are distributed under the same license. 